\title{
Matrix KP: tropical limit and Yang-Baxter maps
}

\author{
Aristophanes Dimakis ${ }^{1}$ - Folkert Müller-Hoissen ${ }^{2}$
}

Received: 8 June 2018 / Revised: 30 August 2018 / Accepted: 3 September 2018 /

Published online: 8 September 2018

(c) The Author(s) 2018

\begin{abstract}
We study soliton solutions of matrix Kadomtsev-Petviashvili (KP) equations in a tropical limit, in which their support at fixed time is a planar graph and polarizations are attached to its constituting lines. There is a subclass of "pure line soliton solutions" for which we find that, in this limit, the distribution of polarizations is fully determined by a Yang-Baxter map. For a vector KP equation, this map is given by an $R$-matrix, whereas it is a nonlinear map in the case of a more general matrix KP equation. We also consider the corresponding Korteweg-deVries reduction. Furthermore, exploiting the fine structure of soliton interactions in the tropical limit, we obtain an apparently new solution of the tetrahedron (or Zamolodchikov) equation. Moreover, a solution of the functional tetrahedron equation arises from the parameter dependence of the vector KP $R$-matrix.
\end{abstract}

Keywords Soliton - KP equation · Yang-Baxter equation · Yang-Baxter map · Tropical limit · Tetrahedron equation

Mathematics Subject Classification 35C08 $\cdot 35 \mathrm{Q} 53 \cdot 37 \mathrm{~K} 10$

\section{Introduction}

A line soliton solution of the scalar Kadomtsev-Petviashvili (KP-II) equation (see, e.g., [16]) is, at fixed time $t$, an exponentially localized wave on a plane. The "tropical limit" takes it to a piecewise linear structure, a planar graph that represents the wave crest, with values of the dependent variable attached to its edges. Via the Maslov dequantization formula (also used in "ultra-discretization" [22]), the tropical limit

$\bowtie$ Folkert Müller-Hoissen

folkert.mueller-hoissen@ds.mpg.de

Aristophanes Dimakis

dimakis@aegean.gr

1 Department of Financial and Management Engineering, University of the Aegean, Chios, Greece

2 Max-Planck-Institute for Dynamics and Self-Organization, Göttingen, Germany 
graph at fixed $t$ can be conveniently computed as the boundary of "dominating phase regions" in the $(x y)$ plane (see Sect. 4). Applications of this method in the context of integrable PDEs can be found in $[3,4,6-8,14,19]$, for example.

In this work, we consider the $m \times n$ matrix potential KP equation

$$
4 \phi_{x t}-\phi_{x x x x}-3 \phi_{y y}-6\left(\phi_{x} K \phi_{x}\right)_{x}+6\left(\phi_{x} K \phi_{y}-\phi_{y} K \phi_{x}\right)=0
$$

where $K$ is a constant $n \times m$ matrix and $\phi$ an $m \times n$ matrix, depending on independent variables $x, y, t$, and a subscript indicates a corresponding partial derivative. We will refer to this equation as $\mathrm{pKP}_{K}$.

If $\phi$ is a solution of (1.1), then $\phi_{R}:=\phi K$ and $\phi_{L}:=K \phi$ solve the ordinary $m \times m$, respectively, $n \times n$, matrix potential $\mathrm{KP}$ equation. We also note that, if $K=T K^{\prime} S$ with a constant $m^{\prime} \times m$ matrix $S$ and a constant $n \times n^{\prime}$ matrix $T$, then the $m^{\prime} \times n^{\prime}$ matrix $\phi^{\prime}=S \phi T$ satisfies the $\mathrm{pKP}_{K^{\prime}}$ equation, as a consequence of (1.1).

In the vector case $n=1$, writing $K=\left(k_{1}, \ldots, k_{m}\right)$ and $\phi=\left(\phi_{1}, \ldots, \phi_{m}\right)^{\top},(1.1)$ becomes the following system of coupled equations,

$$
\begin{aligned}
& 4 \phi_{i, x t}-\phi_{i, x x x x}-3 \phi_{i, y y}-6 \sum_{j=1}^{m} k_{j}\left(\left(\phi_{i, x} \phi_{j, x}\right)_{x}-\phi_{i, x} \phi_{j, y}+\phi_{i, y} \phi_{j, x}\right)=0 \\
& i=1, \ldots, m .
\end{aligned}
$$

By choosing $T=1$ and any invertible $m \times m$ matrix $S$ that has $K$ as its first row, we have $K=K^{\prime} S$ with $K^{\prime}=(1,0, \ldots, 0)$. In terms of the new variable $\phi^{\prime}=S \phi$, the above system thus consists of one scalar pKP equation and $m-1$ linear equations involving the dependent variable of the former.

For

$$
u:=2 \phi_{x}
$$

we obtain from (1.1) the $m \times n$ matrix KP equation

$$
\left(4 u_{t}-u_{x x x}-3(u K u)_{x}\right)_{x}-3 u_{y y}+3\left(u K \int u_{y} \mathrm{~d} x-\int u_{y} \mathrm{~d} x K u\right)_{x}=0 .
$$

The extension of the scalar KP equation to a matrix version achieves that solitons carry internal degrees of freedom. The value of the dependent variable along a segment of the suitably defined (piecewise linear) tropical limit graph will be referred to as "polarization" in the following.

The Korteweg-deVries (KdV) reduction of (1.2) is

$$
4 u_{t}-u_{x x x}-3(u K u)_{x}=0,
$$

which we will refer to as $\mathrm{KdV}_{K}$. If $K$ is the identity matrix, this is the matrix $\mathrm{KdV}$ equation (see, e.g., [11]). The 2 -soliton solution of the latter yields a map from polar- 
izations at $t \ll 0$ to polarizations at $t \gg 0$. It is known [12,24] that this yields a Yang-Baxter map, i.e., a set-theoretical solution of the (quantum) Yang-Baxter equation (also see [1,23] for the case of the vector Nonlinear Schrödinger equation). Not surprisingly, this is a feature preserved in the tropical limit. The surprising new insight, however, is that this map governs the evolution of polarizations throughout the tropical limit graph of a soliton solution. In case of a vector $\mathrm{KdV}$ equation, i.e., $\mathrm{KdV}_{K}$ with $n=1$, it is given by an $R$-matrix, a linear map solution of the Yang-Baxter equation.

More generally, we will explore in this work the tropical limit of "pure" (see Sect. 3) soliton solutions of the above $K$-modified matrix KP equation and demonstrate that a Yang-Baxter map governs their structure. There are lots of soliton solutions beyond pure solitons (see, e.g., [16] for the scalar case), but for them a Yang-Baxter map is no longer sufficient to describe the behavior.

In the case of the vector KP equation, the expression for a pure soliton solution involves a function $\tau$ which is a $\tau$-function of the scalar KP equation. Its tropical limit at fixed $t$ determines a planar graph, and the vector KP soliton solution associates in this limit a constant vector (polarization) with each linear segment of the graph. The polarization values are then related by a linear Yang-Baxter map, represented by an $R$-matrix, which does not depend on the independent variables $x, y, t$, but only on the "spectral parameters" of the soliton solution.

Section 2 summarizes a binary Darboux transformation for the $\mathrm{pKP}_{K}$ equation and applies it to a trivial seed solution in order to obtain soliton solutions. In Sect. 3, we restrict out consideration to the subclass of "pure" soliton solutions. This essentially disregards solutions with substructures of the form of Miles resonances. Section 4 addresses the tropical limit of pure soliton solutions. The cases of two and three solitons are then treated in Sects. 5 and 6. Section 7 provides a general proof of the fact that, in the vector case, an $R$-matrix relates the polarizations at crossings. The linearity of the Yang-Baxter map in the vector case is certainly related to the particularly simple structure of the vector pKP equation mentioned above. In Sect. 8 we show how to construct a pure $N$-soliton solution of the vector KP equation from a pure $N$-soliton solution of the scalar KP equation, $N$ vector data and the aforementioned $R$-matrix. Section 9 extends our exploration of the vector KP 3-soliton case and presents an apparently new solution of the tetrahedron (Zamolodchikov) equation (see, e.g., [9] and references cited there). In Sect. 10, we reveal the structure of the vector KP $R$-matrix, which leads us to a more general two-parameter $R$-matrix. Its parameter dependence determines, via a "local" Yang-Baxter equation [18] (also see [9]), a solution of the functional tetrahedron equation (see, e.g., $[9,15,20]$ ), i.e., the set-theoretical version of the tetrahedron equation. Finally, Sect. 11 contains some concluding remarks.

\section{Soliton solutions of the $K$-modified matrix KP equation}

The following describes a binary Darboux transformation for the $\mathrm{pKP}_{K}$ Eq. (1.1). This is a simple extension of what is presented in [5], for example. Let $\phi_{0}$ be a solution of (1.1). Let $\theta$ and $\chi$ be $m \times N$, respectively, $N \times n$, matrix solutions of the linear equations 


$$
\begin{aligned}
& \theta_{y}=\theta_{x x}+2 \phi_{0, x} K \theta \quad \theta_{t}=\theta_{x x x}+3 \phi_{0, x} K \theta_{x}+\frac{3}{2}\left(\phi_{0, y}+\phi_{0, x x}\right) K \theta, \\
& \chi_{y}=-\chi_{x x}-2 \chi K \phi_{0, x}, \quad \chi_{t}=\chi_{x x x}+3 \chi_{x} K \phi_{0, x}-\frac{3}{2} \chi K\left(\phi_{0, y}-\phi_{0, x x}\right) .
\end{aligned}
$$

Then the system

$$
\begin{aligned}
& \Omega_{x}=-\chi K \theta, \quad \Omega_{y}=-\chi K \theta_{x}+\chi_{x} K \theta, \\
& \Omega_{t}=-\chi K \theta_{x x}+\chi_{x} K \theta_{x}-\chi_{x x} K \theta-3 \chi K \phi_{0, x} K \theta,
\end{aligned}
$$

is compatible and can thus be integrated to yield an $N \times N$ matrix solution $\Omega$. If $\Omega$ is invertible, then

$$
\phi=\phi_{0}-\theta \Omega^{-1} \chi
$$

is a new solution of (1.1).

For vanishing ${ }^{1}$ seed solution, i.e., $\phi_{0}=0$, soliton solutions are obtained as follows. Let

$$
\theta=\sum_{a=1}^{A} \theta_{a} \mathrm{e}^{\vartheta\left(P_{a}\right)}, \quad \chi=\sum_{b=1}^{B} \mathrm{e}^{-\vartheta\left(Q_{b}\right)} \chi_{b},
$$

where $P_{a}, Q_{b}$ are constant $N \times N$ matrices, $\theta_{a}, \chi_{b}$ are constant $m \times N$, respectively, $N \times n$ matrices, and

$$
\vartheta(P)=x P+y P^{2}+t P^{3}
$$

If, for all $a, b$, the matrices $P_{a}$ and $Q_{b}$ have no eigenvalue in common, there are unique $N \times N$ matrix solutions $W_{b a}$ of the Sylvester equations

$$
Q_{b} W_{b a}-W_{b a} P_{a}=\chi_{b} K \theta_{a} \quad a=1, \ldots, A, \quad b=1, \ldots, B .
$$

Then (2.1) is solved by

$$
\Omega=\Omega_{0}+\sum_{a=1}^{A} \sum_{b=1}^{B} \mathrm{e}^{-\vartheta\left(Q_{b}\right)} W_{b a} \mathrm{e}^{\vartheta\left(P_{a}\right)},
$$

with a constant $N \times N$ matrix $\Omega_{0}$, and (2.2) determines a soliton solution of (1.1) [and thus via $u=2 \phi_{x}$ a solution of (1.2)], if $\Omega$ is everywhere invertible.

Remark 2.1 Corresponding solutions of the $\mathrm{pKP}_{K}$ hierarchy are obtained by replacing (2.3) with $\vartheta(P)=\sum_{r=1}^{\infty} t_{r} P^{r}$, where $t_{1}=x, t_{2}=y, t_{3}=t$.

\footnotetext{
${ }_{1}^{1}$ More generally, the following holds for any constant $\phi_{0}$. But adding to $\phi$ a constant matrix is an obvious symmetry of the $\mathrm{pKP}_{K}$ equation. 


\section{Pure soliton solutions}

In the following, we restrict our considerations to the case where $A=B=1$. Then there remains only a single Sylvester equation,

$$
Q_{1} W-W P_{1}=\chi_{1} K \theta_{1}
$$

Moreover, we will restrict the matrices $P_{1}$ and $Q_{1}$ to be diagonal. It is convenient to name the diagonal entries ("spectral parameters") in two different ways,

$$
\begin{aligned}
& P_{1}=\operatorname{diag}\left(p_{1,1}, \ldots, p_{N, 1}\right)=\operatorname{diag}\left(p_{1}, \ldots, p_{N}\right), \\
& Q_{1}=\operatorname{diag}\left(p_{1,2}, \ldots, p_{N, 2}\right)=\operatorname{diag}\left(q_{1}, \ldots, q_{N}\right) .
\end{aligned}
$$

We further write

$$
\theta_{1}=\left(\begin{array}{llll}
\left(q_{1}-p_{1}\right) \xi_{1} & \left(q_{2}-p_{2}\right) \xi_{2} & \cdots & \left(q_{N}-p_{N}\right) \xi_{N}
\end{array}\right), \quad \chi_{1}=\left(\begin{array}{c}
\eta_{1} \\
\vdots \\
\eta_{N}
\end{array}\right)
$$

where $\xi_{i}$ are $m$-component column vectors and $\eta_{i}$ are $n$-component row vectors. Then the solution of the above Sylvester equation is given by

$$
W=\left(w_{i j}\right), \quad w_{i j}=\frac{q_{j}-p_{j}}{q_{i}-p_{j}} \eta_{i} K \xi_{j} \quad i, j=1, \ldots, N
$$

Furthermore, we set $\Omega_{0}=I_{N}$, the $N \times N$ identity matrix. Hence $\Omega=\left(\Omega_{i j}\right)$ with

$$
\Omega_{i j}=\delta_{i j}+w_{i j} \mathrm{e}^{\vartheta\left(p_{j}\right)-\vartheta\left(q_{i}\right)}
$$

where $\delta_{i j}$ is the Kronecker delta. We call soliton solutions obtained from (2.2), with the above restrictions, "pure solitons". All what follows refer to them.

We introduce

$$
\vartheta_{I}:=\sum_{i=1}^{N} \vartheta\left(p_{i, a_{i}}\right) \text { if } I=\left(a_{1}, \ldots, a_{N}\right) \in\{1,2\}^{N} .
$$

Instead of using $\left(a_{1}, \ldots, a_{N}\right)$ as a subscript (or superscript), we will simply write $a_{1} \ldots a_{N}$ in the following. For example, $\vartheta_{a_{1} \ldots a_{N}}=\vartheta_{\left(a_{1}, \ldots, a_{N}\right)}$.

From (2.2), we find that the pure soliton solutions of the $\mathrm{pKP}_{K}$ equation are given by

$$
\phi=\frac{F}{\tau},
$$


with

$$
\begin{aligned}
\tau & :=\mathrm{e}^{\vartheta_{2}} \operatorname{det} \Omega, \\
F & :=-\mathrm{e}^{\vartheta_{2}} \theta_{1} \mathrm{e}^{\vartheta\left(P_{1}\right)} \operatorname{adj}(\Omega) \mathrm{e}^{-\vartheta\left(Q_{1}\right)} \chi_{1},
\end{aligned}
$$

where $\operatorname{adj}(\Omega)$ denotes the adjugate of the matrix $\Omega$ and $2:=2 \ldots 2=(2, \ldots, 2)$.

Proposition $3.1 \tau$ and $F$ have expansions

$$
\begin{aligned}
\tau & =\sum_{I \in\{1,2\}^{N}} \mu_{I} e^{\vartheta_{I}}, \\
F & =\sum_{I \in\{1,2\}^{N}} M_{I} e^{\vartheta_{I}},
\end{aligned}
$$

with constants $\mu_{I}$ and constant $m \times n$ matrices $M_{I}$, where $\mu_{2}=1$ and $M_{2}=0$.

Proof From the definition of the determinant, det $\Omega=\epsilon^{i_{1} \ldots i_{N}} \Omega_{1 i_{1}} \cdots \Omega_{N i_{N}}$, with the Levi-Civita symbol $\epsilon^{i_{1} \ldots i_{N}}$ and summation convention, we know that det $\Omega$ consists of a sum of monomials of order $N$ in the entries $\Omega_{i j}$. Here the latter is given by (3.1). If no diagonal term $\Omega_{i i}$ appears in a monomial, its phase factor is $\mathrm{e}^{\vartheta_{\mathbf{1}}-\vartheta_{\mathbf{2}}}$. If one diagonal entry $\Omega_{i i}=1+w_{i i} \mathrm{e}^{\vartheta\left(p_{i, 1}\right)-\vartheta\left(p_{i, 2}\right)}$ appears in a monomial, the latter splits into two parts. Only the part arising from the summand 1 is different as now the phase factor is $\mathrm{e}^{\vartheta_{1}-\vartheta_{\mathbf{2}}-\vartheta\left(p_{i, 1}\right)+\vartheta\left(p_{i, 2}\right)}$. From monomials containing several diagonal entries of $\Omega$, we obtain summands with a phase factor of the form

$$
\begin{aligned}
& \mathrm{e}^{\vartheta \vartheta_{\mathbf{1}}-\vartheta_{\mathbf{2}}-\vartheta\left(p_{i_{1}, 1}\right)+\vartheta\left(p_{i_{1}, 2}\right)+\cdots-\vartheta\left(p_{i_{r}, 1}\right)+\vartheta\left(p_{i_{r}, 2}\right)} \\
& \quad=\mathrm{e}^{\left[\vartheta_{\mathbf{1}}-\vartheta\left(p_{i_{1}, 1}\right)-\cdots-\vartheta\left(p_{i_{r}, 1}\right)\right]+\vartheta\left(p_{i_{1}, 2}\right)+\cdots+\vartheta\left(p_{i_{r}, 2}\right)-\vartheta_{\mathbf{2}}} .
\end{aligned}
$$

Finally, from a monomial with $N$ diagonal entries of $\Omega$, we also obtain a constant term, namely 1 . Now our assertion (3.5) follows since $\tau$ is $\operatorname{det} \Omega$ multiplied by $\mathrm{e}^{\vartheta_{2}}$. Clearly, $\mu_{2}=1$.

According to the Laplace (cofactor) expansion det $\Omega=\sum_{j=1}^{N} \Omega_{i j} \operatorname{adj}(\Omega)_{j i}$ with respect to the $i$ th row, the term $\Omega_{i j} \operatorname{adj}(\Omega)_{j i}$ consists of all summands in det $\Omega$ having $\Omega_{i j}$ as a factor. (3.5) implies that a summand of $\mathrm{e}^{\vartheta_{2}} \operatorname{adj}(\Omega)_{j i}$ then has a phase factor of the form $\mathrm{e}^{\vartheta_{I}-\vartheta\left(p_{j, 1}\right)+\vartheta\left(p_{i, 2}\right)}$, with some $I \in\{1,2\}^{N}$, so that $\mathrm{e}^{\vartheta 2}\left(\mathrm{e}^{\vartheta\left(P_{1}\right)} \operatorname{adj}(\Omega) \mathrm{e}^{-\vartheta\left(Q_{1}\right)}\right)_{j i}$ has the phase factor $\mathrm{e}^{\vartheta_{I}}$. Hence (3.6) holds. Furthermore, no entry of $\mathrm{e}^{\vartheta\left(P_{1}\right)} \operatorname{adj}(\Omega) \mathrm{e}^{-\vartheta\left(Q_{1}\right)}$ is constant, and hence, $M_{\mathbf{2}}=0$.

Remark 3.2 The introduction of the redundant factor $\mathrm{e}^{\vartheta_{2}}$ in (3.2), via the definitions (3.3) and (3.4), achieves that $\tau$ and $F$ are linear combinations of exponentials $\mathrm{e}^{\vartheta_{I}}$, $I \in\{1,2\}^{N}$, in which case we have a very convenient labeling. This is also so if we choose the factor $\mathrm{e}^{-\vartheta_{1}}$ instead, which leads to an expansion in terms of $\mathrm{e}^{-\vartheta_{I}}$, now with $M_{1}=0$.

Regularity of a pure soliton solution requires $\mu_{I} \geq 0$ for all $I \in\{1,2\}^{N}$ (or equivalently $\mu_{I} \leq 0$ for all $I \in\{1,2\}^{N}$ ) and $\mu_{I} \neq 0$ for at least one $I$. If $\mu_{I}=0$ for 
some $I$, this means that the phase $\vartheta_{I}$ is not present in the expression for $\tau$. In this case, one has to arrange the data in such a way that $M_{I}=0$ in order to avoid unbounded exponential growth of the soliton solution in some phase region. But we will disregard such cases and add the condition $\mu_{I}>0, \forall I \in\{1,2\}^{N}$, to our definition of pure soliton solutions.

It follows that the corresponding solution of the KP equation is given by

$$
u=\frac{1}{\tau^{2}} \sum_{I, J \in\{1,2\}^{N}}\left(p_{J}-p_{I}\right)\left(\mu_{I} M_{J}-\mu_{J} M_{I}\right) \mathrm{e}^{\vartheta_{I}} \mathrm{e}^{\vartheta_{J}},
$$

where

$$
p_{I}=p_{1, a_{1}}+\cdots+p_{N, a_{N}} \quad \text { if } \quad I=\left(a_{1}, \ldots, a_{N}\right) .
$$

Using Jacobi's formula for the derivative of a determinant, we obtain

$$
\begin{aligned}
\tau_{x} & =p_{2} \tau+\mathrm{e}^{\vartheta_{2}} \operatorname{tr}\left(\operatorname{adj}(\Omega) \Omega_{x}\right) \\
& =p_{2} \tau-\mathrm{e}^{\vartheta_{2}} \operatorname{tr}(\operatorname{adj}(\Omega) \chi K \theta)=p_{2} \tau-\mathrm{e}^{\vartheta_{2}} \operatorname{tr}(K \theta \operatorname{adj}(\Omega) \chi) \\
& =p_{2} \tau+\operatorname{tr}(K F)
\end{aligned}
$$

which implies

$$
\operatorname{tr}(K \phi)=(\ln \tau)_{x}-p_{2},
$$

and thus

$$
\operatorname{tr}(K u)=2(\ln \tau)_{x x}
$$

Using (3.2) in (3.7), and reading off the coefficient of $\mathrm{e}^{\vartheta_{I}}$, we find

$$
\operatorname{tr}\left(K M_{I}\right)=\left(p_{I}-p_{2}\right) \mu_{I}
$$

Remark 3.3 If $n=1$, (3.7) reads

$$
K \phi=(\ln \tau)_{x}-p_{2},
$$

and

$$
K u=2(\ln \tau)_{x x}
$$

is a solution of the scalar KP equation. If $n>1, \operatorname{tr}(K u)$ is not in general a solution of the scalar KP equation. 
Remark 3.4 Dropping the redundant factor $\mathrm{e}^{\vartheta_{2}}$ in (3.3) and (3.4) means that we have to multiply the above expressions (3.5) and (3.6) for $\tau$ and $F$ by $\mathrm{e}^{-\vartheta_{2}}$. It is then evident that $\phi$ only depends on differences of phases of the form $\vartheta\left(p_{i, 1}\right)-\vartheta\left(p_{i, 2}\right)=$ $\left(p_{i, 1}-p_{i, 2}\right) x+\left(p_{i, 1}^{2}-p_{i, 2}^{2}\right) y+\left(p_{i, 1}^{3}-p_{i, 2}^{3}\right) t$. As a consequence, setting $p_{i, 2}=-p_{i, 1}$, i.e., $q_{i}=-p_{i}$, eliminates the $y$ terms in all phases. This means that under this condition for the parameters, we could have started as well with $\vartheta(P)=x P+t P^{3}$, hence without the $y$ term in (2.3). In this way, contact is made with the $\mathrm{KdV}_{K}$ reduction in $\mathrm{KP}_{K}$.

\section{Tropical limit of pure soliton solutions}

A crucial point is that we define the tropical limit of the matrix soliton solution via the tropical limit of the scalar function $\tau$ (cf. [6-8]). Let

$$
\phi_{I}:=\left.\phi\right|_{\vartheta_{J} \rightarrow-\infty, J \neq I}=\frac{M_{I}}{\mu_{I}} .
$$

In a region where a phase $\vartheta_{I}$ dominates all others, in the sense that $\log \left(\mu_{I} \mathrm{e}^{\vartheta_{I}}\right)>$ $\log \left(\mu_{J} \mathrm{e}^{\vartheta_{J}}\right)$ for all participating $J \neq I$, the tropical limit of the potential $\phi$ is given by (4.1). It should be noticed that these expressions do not depend on the coordinates $x, y, t$.

The boundary between the regions associated with the phases $\vartheta_{I}$ and $\vartheta_{J}$ is determined by the condition

$$
\mu_{I} \mathrm{e}^{\vartheta_{I}}=\mu_{J} \mathrm{e}^{\vartheta_{J}}
$$

Not all parts of such a boundary are visible at fixed time, since some of them may lie in a region where a third phase dominates the two phases. The tropical limit of a soliton solution at a fixed time $t$ has support on the visible parts of the boundaries between the regions associated with phases appearing in $\tau$. On such a visible boundary segment, the value of $u$ is given by

$$
u_{I J}=\frac{1}{2}\left(p_{I}-p_{J}\right)\left(\phi_{I}-\phi_{J}\right)
$$

For $I=\left(a_{1}, \ldots, a_{N}\right)$ we set

$$
I_{k}(a)=\left(a_{1}, \ldots, a_{k-1}, a, a_{k+1}, \ldots, a_{N}\right)
$$

At fixed time, the set of line segments associated with the $k$ th soliton are obtained from (4.2) with $I=I_{k}(1)$ and $J=I_{k}(2)$, for all possible $I$. They satisfy

$$
\left(p_{k, 2}-p_{k, 1}\right) x+\left(p_{k, 2}^{2}-p_{k, 1}^{2}\right) y+\left(p_{k, 2}^{3}-p_{k, 1}^{3}\right) t+\ln \frac{\mu_{I_{k}(2)}}{\mu_{I_{k}(1)}}=0 .
$$


All these line segments have the same slope $-\left(p_{k, 2}+p_{k, 1}\right)^{-1}$ in the $x y$ plane, and hence, they are parallel. The shifts between them are given by

$$
\delta_{I J}^{(k)}=\ln \left(\frac{\mu_{I_{k}(2)}}{\mu_{I_{k}(1)}} \frac{\mu_{J_{k}(1)}}{\mu_{J_{k}(2)}}\right) \text {. }
$$

They give rise to the familiar asymptotic "phase shifts" of line solitons. The tropical limit of $u$ on a visible line segment of the $k$ th soliton is given by

$$
u_{I_{k}(1) I_{k}(2)}=\frac{1}{2}\left(p_{k, 1}-p_{k, 2}\right)\left(\phi_{I_{k}(1)}-\phi_{I_{k}(2)}\right) \text {. }
$$

The value of $u$ at a visible triple phase coincidence is

$$
\begin{aligned}
u_{I J L}= & \frac{4}{9}\left(u_{I J}+u_{I L}+u_{J L}\right)=\frac{2}{9}\left(\left(2 p_{I}-p_{J}-p_{L}\right) \phi_{I}+\left(2 p_{J}-p_{I}-p_{L}\right) \phi_{J}\right. \\
& \left.+\left(2 p_{L}-p_{I}-p_{J}\right) \phi_{L}\right) .
\end{aligned}
$$

Instead of the above expressions for the tropical values of $u$ we will rather consider

$$
\hat{u}_{I J}=\frac{\phi_{I}-\phi_{J}}{p_{I}-p_{J}},
$$

which has the form of a discrete derivative. Since (3.8) and (4.1) imply

$$
\operatorname{tr}\left(K \phi_{I}\right)=p_{I}-p_{2}
$$

the latter values are normalized in the sense that

$$
\operatorname{tr}\left(K \hat{u}_{I J}\right)=1
$$

If $I=\left(a_{1}, \ldots, a_{N}\right)$ and $i \neq j$, let

$$
I_{i j}(a, b)=\left.\left(a_{1}, \ldots, a_{N}\right)\right|_{a_{i} \mapsto a, a_{j} \mapsto b} .
$$

The normalized tropical values of $u$ satisfy

$$
\begin{aligned}
& \left(p_{i}-q_{i}\right) \hat{u}_{I_{i j}(1,1) I_{i j}(2,1)}+\left(p_{j}-q_{j}\right) \hat{u}_{I_{i j}(2,1) I_{i j}(2,2)}=\left(p_{i}-q_{i}+p_{j}-q_{j}\right) \hat{u}_{I_{i j}(1,1) I_{i j}(2,2),}, \\
& \left(p_{i}-q_{i}\right) \hat{u}_{I_{i j}(1,2) I_{i j}(2,2)}+\left(p_{j}-q_{j}\right) \hat{u}_{I_{i j}(1,1) I_{i j}(1,2)}=\left(p_{i}-q_{i}+p_{j}-q_{j}\right) \hat{u}_{I_{i j}(1,1) I_{i j}(2,2),}, \\
& \left(p_{i}-q_{i}\right) \hat{u}_{I_{i j}(1,1) I_{i j}(2,1)}+\left(p_{j}-q_{j}\right) \hat{u}_{I_{i j}(1,1), I_{i j}(1,2)}=\left(p_{i}-q_{i}+q_{j}-p_{j}\right) \hat{u}_{I_{i j}(1,2) I_{i j}(2,1)}, \\
& \left(p_{i}-q_{i}\right) \hat{u}_{I_{i j}(1,2) I_{i j}(2,2)}+\left(p_{j}-q_{j}\right) \hat{u}_{I_{i j}(2,1), I_{i j}(2,2)}=\left(p_{i}-q_{i}+q_{j}-p_{j}\right) \hat{u}_{I_{i j}(1,2) I_{i j}(2,1)} .
\end{aligned}
$$

These identities are simply consequences of the definition of $\hat{u}_{I J}$. They linearly relate the (normalized) polarizations at points of the tropical limit graph, where three lines meet. 


\section{Pure 2-soliton solutions}

Let $N=2$. Then we have

$$
\tau=\mathrm{e}^{\vartheta_{22}}+\kappa_{11} \mathrm{e}^{\vartheta_{12}}+\kappa_{22} \mathrm{e}^{\vartheta_{21}}+\alpha \kappa_{11} \kappa_{22} \mathrm{e}^{\vartheta_{11}}
$$

where

$$
\kappa_{i j}=\eta_{i} K \xi_{j}, \alpha=1-\frac{\left(q_{1}-p_{1}\right)\left(q_{2}-p_{2}\right) \kappa_{12} \kappa_{21}}{\left(q_{1}-p_{2}\right)\left(q_{2}-p_{1}\right) \kappa_{11} \kappa_{22}},
$$

and

$$
\begin{aligned}
F= & \left(q_{1}-p_{1}\right)\left(q_{2}-p_{2}\right)\left(\frac{\kappa_{22}}{p_{2}-q_{2}} \xi_{1} \otimes \eta_{1}+\frac{\kappa_{11}}{p_{1}-q_{1}} \xi_{2} \otimes \eta_{2}+\frac{\kappa_{12}}{q_{1}-p_{2}} \xi_{1} \otimes \eta_{2}\right. \\
& \left.+\frac{\kappa_{21}}{q_{2}-p_{1}} \xi_{2} \otimes \eta_{1}\right) \mathrm{e}^{\vartheta_{11}}+\left(p_{1}-q_{1}\right) \xi_{1} \otimes \eta_{1} \mathrm{e}^{\vartheta_{12}}+\left(p_{2}-q_{2}\right) \xi_{2} \otimes \eta_{2} \mathrm{e}^{\vartheta_{21}}
\end{aligned}
$$

The tropical values of the $\mathrm{pKP}_{K}$ solution $\phi$ in the dominant phase regions are then given by

$$
\begin{aligned}
& \phi_{11}= \frac{\left(q_{1}-p_{1}\right)\left(q_{2}-p_{2}\right)}{\alpha \kappa_{11} \kappa_{22}}\left(\frac{\kappa_{22}}{p_{2}-q_{2}} \xi_{1} \otimes \eta_{1}+\frac{\kappa_{11}}{p_{1}-q_{1}} \xi_{2} \otimes \eta_{2}\right. \\
&\left.\quad+\frac{\kappa_{12}}{q_{1}-p_{2}} \xi_{1} \otimes \eta_{2}+\frac{\kappa_{21}}{q_{2}-p_{1}} \xi_{2} \otimes \eta_{1}\right), \\
& \phi_{12}=\left(p_{1}-q_{1}\right) \frac{\xi_{1} \otimes \eta_{1}}{\kappa_{11}}, \quad \phi_{21}=\left(p_{2}-q_{2}\right) \frac{\xi_{2} \otimes \eta_{2}}{\kappa_{22}}, \quad \phi_{22}=0 .
\end{aligned}
$$

Remark 5.1 The above values $\phi_{a b}$ solve the following nonlinear equation,

$$
\begin{gathered}
\left(1+\operatorname{tr} \frac{K\left(\phi_{12}-\phi_{22}\right) K\left(\phi_{21}-\phi_{22}\right)}{\left(q_{1}-p_{2}\right)\left(p_{1}-q_{2}\right)}\right)\left(\phi_{11}-\phi_{22}\right)-\frac{\left(\phi_{12}-\phi_{22}\right) K\left(\phi_{21}-\phi_{22}\right)}{q_{1}-p_{2}} \\
+\frac{\left(\phi_{21}-\phi_{22}\right) K\left(\phi_{12}-\phi_{22}\right)}{p_{1}-q_{2}}-\left(\phi_{12}-\phi_{22}\right)-\left(\phi_{21}-\phi_{22}\right)=0 .
\end{gathered}
$$

Addressing more than two solitons, nonzero counterparts of $\phi_{22}$ will show up, as displayed in this equation.

For the tropical values of $\hat{u}$ along the phase region boundaries, we obtain

$$
\begin{aligned}
& u_{1, \text { in }}:=\hat{u}_{11,21}=\alpha^{-1}\left(1_{m}-\frac{q_{2}-p_{2}}{q_{2}-p_{1}} \frac{\xi_{2} \otimes \eta_{2}}{\kappa_{22}} K\right) \frac{\xi_{1} \otimes \eta_{1}}{\kappa_{11}}\left(1_{n}-\frac{q_{2}-p_{2}}{q_{1}-p_{2}} K \frac{\xi_{2} \otimes \eta_{2}}{\kappa_{22}}\right), \\
& u_{2, \text { in }}:=\hat{u}_{21,22}=\frac{\xi_{2} \otimes \eta_{2}}{\kappa_{22}} \\
& u_{1, \text { out }}:=\hat{u}_{12,22}=\frac{\xi_{1} \otimes \eta_{1}}{\kappa_{11}},
\end{aligned}
$$




$$
u_{2, \text { out }}:=\hat{u}_{11,12}=\alpha^{-1}\left(1_{m}-\frac{q_{1}-p_{1}}{q_{1}-p_{2}} \frac{\xi_{1} \otimes \eta_{1}}{\kappa_{11}} K\right) \frac{\xi_{2} \otimes \eta_{2}}{\kappa_{22}}\left(1_{n}-\frac{q_{1}-p_{1}}{q_{2}-p_{1}} K \frac{\xi_{1} \otimes \eta_{1}}{\kappa_{11}}\right),
$$

where $1_{m}$ stands for the $m \times m$ identity matrix. For the in/out classification, see Fig. 1 below. All the matrices in (5.2) have rank one, which is not at all obvious from the form of $\phi_{a b}$. We obtain the following nonlinear relation between "incoming" and "outgoing" polarizations,

$$
\begin{aligned}
& u_{1, \text { out }}=\alpha_{\text {in }}^{-1}\left(1_{m}-\frac{q_{2}-p_{2}}{p_{1}-p_{2}} u_{2, \text { in }} K\right) u_{1, \text { in }}\left(1_{n}-\frac{p_{2}-q_{2}}{q_{1}-q_{2}} K u_{2, \text { in }}\right), \\
& u_{2, \text { out }}=\alpha_{\text {in }}^{-1}\left(1_{m}-\frac{q_{1}-p_{1}}{q_{1}-q_{2}} u_{1, \text { in }} K\right) u_{2, \text { in }}\left(1_{n}-\frac{p_{1}-q_{1}}{p_{1}-p_{2}} K u_{1, \text { in }}\right),
\end{aligned}
$$

where

$$
\alpha_{\text {in }}=1-\frac{\left(p_{1}-q_{1}\right)\left(p_{2}-q_{2}\right)}{\left(p_{1}-p_{2}\right)\left(q_{1}-q_{2}\right)} \operatorname{tr}\left(K u_{1, \text { in }} K u_{2, \text { in }}\right) .
$$

We note that $\alpha \alpha_{\text {in }}=1$. (5.3) determines a new nonlinear Yang-Baxter map

$$
\mathcal{R}: \quad\left(u_{1, \text { in }}, u_{2, \text { in }}\right) \mapsto\left(u_{1, \text { out }}, u_{2, \text { out }}\right),
$$

with parameters $p_{i}, q_{i}, i=1,2$. We verified directly that this satisfies the Yang-Baxter equation

$$
\mathcal{R}_{12} \circ \mathcal{R}_{13} \circ \mathcal{R}_{23}=\mathcal{R}_{23} \circ \mathcal{R}_{13} \circ \mathcal{R}_{12},
$$

where the subscripts indicate on which two factors of a threefold Cartesian product the map $\mathcal{R}$ acts. An explanation why the Yang-Baxter equation holds will be provided in Sect. 6.

Writing

$$
u_{a, \text { in }}=\frac{\xi_{a, \text { in }} \otimes \eta_{a, \text { in }}}{\eta_{a, \text { in }} K \xi_{a, \text { in }}}, \quad u_{a, \text { out }}=\frac{\xi_{a, \text { out }} \otimes \eta_{a, \text { out }}}{\eta_{a, \text { out }} K \xi_{a, \text { out }}} \quad a=1,2,
$$

determines $\xi_{1 \text {,in/out }}$ and $\eta_{1 \text {,in/out up to scalings. We find }}$

$$
\begin{aligned}
& \xi_{1, \text { out }}=\alpha_{\text {in }}^{-1 / 2}\left(1_{m}-\frac{p_{2}-q_{2}}{p_{2}-p_{1}} \frac{\xi_{2, \text { in }} \otimes \eta_{2, \text { in }}}{\eta_{2, \text { in }} K \xi_{2, \text { in }}} K\right) \xi_{1, \text { in }}, \\
& \xi_{2, \text { out }}=\alpha_{\text {in }}^{-1 / 2}\left(1_{m}-\frac{q_{1}-p_{1}}{q_{1}-q_{2}} \frac{\xi_{1, \text { in }} \otimes \eta_{1, \text { in }}}{\eta_{1, \text { in }} K \xi_{1, \text { in }}} K\right) \xi_{2, \text { in }}, \\
& \eta_{1, \text { out }}=\alpha_{\text {in }}^{-1 / 2} \eta_{1, \text { in }}\left(1_{n}-\frac{q_{2}-p_{2}}{q_{2}-q_{1}} K \frac{\xi_{2, \text { in }} \otimes \eta_{2, \text { in }}}{\eta_{2, \text { in }} K \xi_{2, \text { in }}}\right),
\end{aligned}
$$



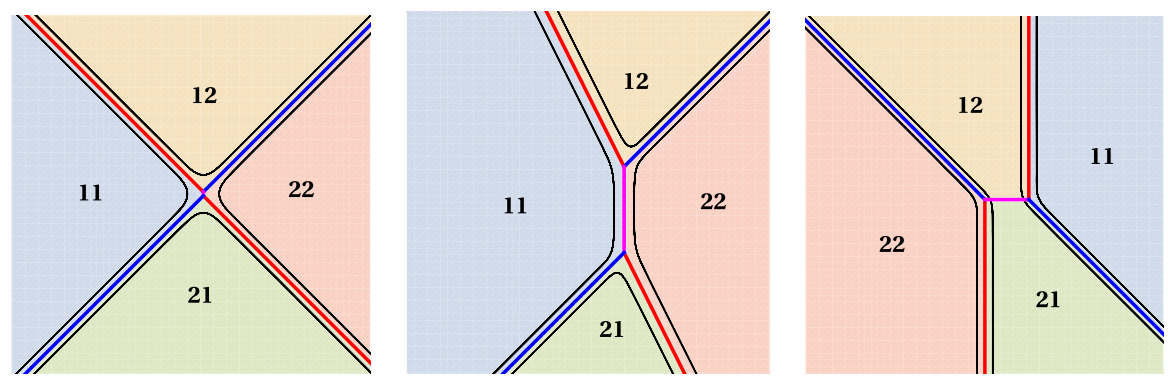

Fig. 1 The first is a contour plot of $\operatorname{tr}(K u)=2(\ln \tau)_{x x}$ for a 2-soliton solution of the $2 \times 3$ matrix KP equation, at $t=0$ in the $x y$ plane, using the data of Example 5.3. Viewed as a process in $y$ direction, the YB map takes the values of the KP variable on the lower two legs to those on the upper two. A number $i j$ indicates the respective dominating phase region. In the second plot, the value of $p_{2}$ is replaced by $-1 / 4+10^{-5}$, so that $p_{2}$ is very close to $q_{1}$. Here, a boundary segment between phase regions 11 and 22 is visible. The third plot presents an example, where the parameters of the 2-soliton solution are now chosen such that the latter boundary is hidden and instead a boundary segment between phase regions 12 and 21 is visible

$$
\eta_{2, \text { out }}=\alpha_{\text {in }}^{-1 / 2} \eta_{2, \text { in }}\left(1_{n}-\frac{p_{1}-q_{1}}{p_{1}-p_{2}} K \frac{\xi_{1, \text { in }} \otimes \eta_{1, \text { in }}}{\eta_{1, \text { in }} K \xi_{1, \text { in }}}\right),
$$

and

$$
\left(\xi_{1, \text { in }}, \eta_{1, \text { in }} ; \xi_{2, \text { in }}, \eta_{2, \text { in }}\right) \mapsto\left(\xi_{1, \text { out }}, \eta_{1, \text { out }} ; \xi_{2, \text { out }}, \eta_{2, \text { out }}\right)
$$

is another form of the above Yang-Baxter map.

Remark 5.2 In (5.2), we found that $u_{2}$,in and $u_{1 \text {,out }}$ have a simple elementary form. They are the polarizations at the two boundary lines of the dominating phase region numbered by $22=(2,2)$, see Fig. 1 . We know from Proposition 3.1 that it is special since $M_{22}=0$. Considering an "evolution" in negative $x$ direction (instead of $y$ direction), thus, offers a more direct derivation of the Yang-Baxter map.

Example 5.3 Let $m=3$ and $n=2$. Choosing

$$
p_{1}=-3 / 4, \quad p_{2}=1 / 4, \quad q_{1}=-1 / 4, \quad q_{2}=3 / 4,
$$

and

$\eta_{1}=(1,0), \quad \eta_{2}=(0,1), \quad \xi_{1}=\left(\begin{array}{c}2 / 3 \\ 5 \\ -2\end{array}\right), \quad \xi_{2}=\left(\begin{array}{c}1 \\ 2 / 3 \\ 2\end{array}\right), \quad K=\left(\begin{array}{lll}1 & 1 & 1 \\ 1 & 2 & 1\end{array}\right)$,

we obtain the first contour plot, at $t=0$, shown in Fig. 1. Figure 2 shows plots of the components of the transpose of $u$. Choosing instead $p_{2}$ close to $q_{1}$ reveals an "inner structure" of crossings, see the second plot in Fig. 1. This is the (phase) shift mentioned in Sect. 4. 

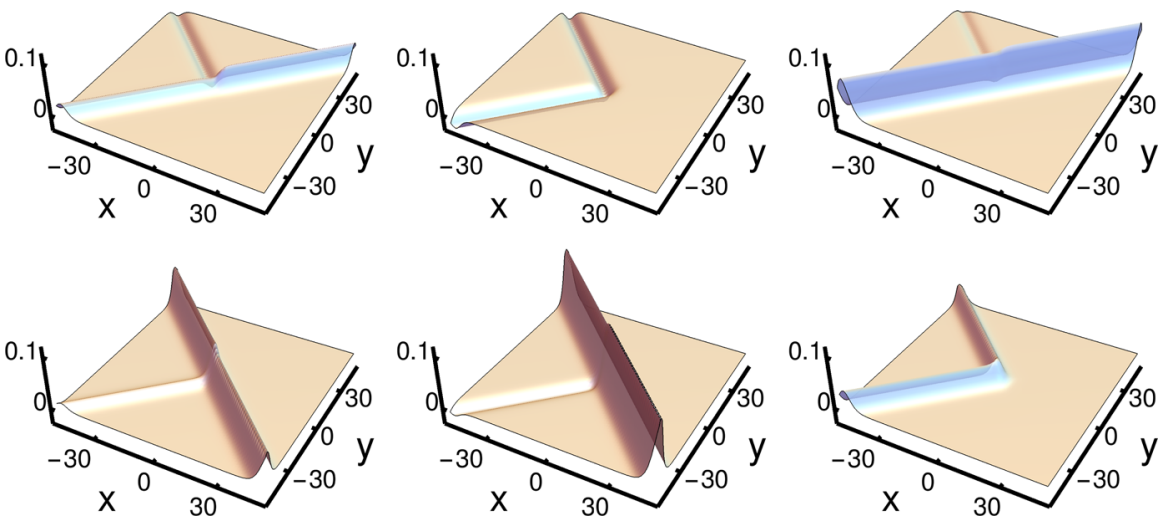

Fig. 2 Plot of the six components of the transpose of $u$ at $t=0$ for the solution of the $3 \times 2$ matrix KP equation with the (first) data specified in Example 5.3. The components are localized exactly where $\operatorname{tr}(K u)$ is localized, cf. Fig. 1

Remark 5.4 We should stress that the relevant structures are actually three-dimensional and our figures only display a two-dimensional cross section. Instead of displaying structures in the $x y$ plane at constant $t$, we may as well look at those in the $x t$ plane at constant $y$. The latter becomes relevant if we consider the $\mathrm{KdV}_{K}$ reduction.

Remark 5.5 Soliton solutions of $\mathrm{KdV}_{K}$ are obtained from those of $\mathrm{KP}_{K}$ by setting $q_{i}=-p_{i}, i=1, \ldots, N$, see Remark 3.4. Then the above equations reduce to

$$
\begin{aligned}
\xi_{1, \text { out }} & =\alpha_{\text {in }}^{-1 / 2}\left(1_{m}-\frac{2 p_{2}}{p_{2}-p_{1}} \frac{\xi_{2, \text { in }} \otimes \eta_{2, \text { in }}}{\eta_{2, \text { in }} K \xi_{2, \text { in }}} K\right) \xi_{1, \text { in }}, \\
\xi_{2, \text { out }} & =\alpha_{\text {in }}^{-1 / 2}\left(1_{m}-\frac{2 p_{1}}{p_{1}-p_{2}} \frac{\xi_{1, \text { in }} \otimes \eta_{1, \text { in }}}{\eta_{1, \text { in }} K \xi_{1, \text { in }}} K\right) \xi_{2, \text { in }}, \\
\eta_{1, \text { out }} & =\alpha_{\text {in }}^{-1 / 2} \eta_{1, \text { in }}\left(1_{n}-\frac{2 p_{2}}{p_{2}-p_{1}} K \frac{\xi_{2, \text { in }} \otimes \eta_{2, \text { in }}}{\eta_{2, \text { in }} K \xi_{2, \text { in }}}\right), \\
\eta_{2, \text { out }} & =\alpha_{\text {in }}^{-1 / 2} \eta_{2, \text { in }}\left(1_{n}-\frac{2 p_{1}}{p_{1}-p_{2}} K \frac{\xi_{1, \text { in }} \otimes \eta_{1, \text { in }}}{\eta_{1, \text { in }} K \xi_{1, \text { in }}}\right),
\end{aligned}
$$

with

$$
\alpha_{\text {in }}=1+\frac{4 p_{1} p_{2}}{\left(p_{1}-p_{2}\right)^{2}} \frac{\eta_{1, \text { in }} K \xi_{2, \text { in }} \eta_{2, \text { in }} K \xi_{1, \text { in }}}{\eta_{1, \text { in }} K \xi_{1, \text { in }} \eta_{2, \text { in }} K \xi_{2, \text { in }}} .
$$

If $K$ is the $N \times N$ identity matrix, this becomes the Yang-Baxter map first found by Veselov [24], also see [12,21]. The factor $\alpha_{\text {in }}^{-1 / 2}$ is missing in these publications, but such a factor is necessary for the map to satisfy the Yang-Baxter equation. It should be noticed that Veselov's "Lax pair" [24] only determines the Yang-Baxter map up to a factor, but such a factor has to be chosen appropriately in order to satisfy the Yang-Baxter equation. One can avoid the square root in the above expression at the price of having an asymmetric appearance of factors $\alpha_{\text {in }}^{-1}$. 


\subsection{Pure column vector 2-soliton solutions}

We set $n=1$. Now the $\eta_{i}$ are scalars and drop out of the relevant formulas. Introducing

$$
\hat{\xi}_{i}=\frac{\xi_{i}}{K \xi_{i}}
$$

we have

$$
\begin{aligned}
u_{1, \text { in }} & =\frac{p_{1}-q_{2}}{p_{1}-p_{2}} \hat{\xi}_{1}+\frac{q_{2}-p_{2}}{p_{1}-p_{2}} \hat{\xi}_{2}, \quad u_{2, \text { in }}=\hat{\xi}_{2}, \\
u_{1, \text { out }} & =\hat{\xi}_{1}, \quad u_{2, \text { out }}=\frac{p_{1}-q_{1}}{p_{1}-p_{2}} \hat{\xi}_{1}+\frac{q_{1}-p_{2}}{p_{1}-p_{2}} \hat{\xi}_{2},
\end{aligned}
$$

and thus

$$
\left(u_{1, \text { out }}, u_{2, \text { out }}\right)=\left(u_{1, \text { in }}, u_{2, \text { in }}\right)\left(\begin{array}{ll}
\frac{p_{1}-p_{2}}{p_{1}-q_{2}} & \frac{p_{1}-q_{1}}{p_{1}-q_{2}} \\
\frac{p_{2}-q_{2}}{p_{1}-q_{2}} & \frac{q_{1}-q_{2}}{p_{1}-q_{2}}
\end{array}\right) .
$$

Generalizing the matrix that appears on the right-hand side to

$$
R\left(p_{i}, q_{i} ; p_{j}, q_{j}\right)=\left(\begin{array}{ll}
\frac{p_{i}-p_{j}}{p_{i}-q_{j}} & \frac{p_{i}-q_{i}}{p_{i}-q_{j}} \\
\frac{p_{j}-q_{j}}{p_{i}-q_{j}} & \frac{q_{i}-q_{j}}{p_{i}-q_{j}}
\end{array}\right),
$$

and letting this act from the right on the $i$ th and $j$ th slot of a threefold direct sum, the Yang-Baxter equation holds. This can be checked directly or inferred from a 3-soliton solution, see Sect. 6.

Remark 5.6 The reduction to vector $\mathrm{KdV}_{K}$ via $q_{i}=-p_{i}$ (see Remark 3.4) leads to

$$
R\left(p_{i}, p_{j}\right)=\left(\begin{array}{ll}
\frac{p_{i}-p_{j}}{p_{i}+p_{j}} & \frac{2 p_{i}}{p_{i}+p_{j}} \\
\frac{2 p_{j}}{p_{i}+p_{j}} & \frac{p_{j}-p_{i}}{p_{i}+p_{j}}
\end{array}\right)
$$

This rules the evolution of initial polarizations (at $t \ll 0$ ) step by step along the tropical limit graph in two-dimensional space-time. The $R$-matrix (5.7) also describes the elastic collision of non-relativistic particles with masses $p_{i}$ in one dimension, see [17].

\subsection{Pure row vector 2-soliton solutions}

Now we set $m=1$. Then the $\xi_{i}$ are scalars and drop out of the relevant formulas. Introducing

$$
\hat{\eta}_{i}=\frac{\eta_{i}}{\eta_{i} K}
$$


we have

$$
\begin{aligned}
u_{1, \text { in }} & =\frac{q_{1}-p_{2}}{q_{1}-q_{2}} \hat{\eta}_{1}+\frac{p_{2}-q_{2}}{q_{1}-q_{2}} \hat{\eta}_{2}, \quad u_{2, \text { in }}=\hat{\eta}_{2}, \\
u_{1, \text { out }} & =\hat{\eta}_{1}, \quad u_{2, \text { out }}=\frac{q_{1}-p_{1}}{q_{1}-q_{2}} \hat{\eta}_{1}+\frac{p_{1}-q_{2}}{q_{1}-q_{2}} \hat{\eta}_{2},
\end{aligned}
$$

so that

$$
\left(\begin{array}{l}
u_{1, \text { out }} \\
u_{2, \text { out }}
\end{array}\right)=\left(\begin{array}{ll}
\frac{q_{2}-q_{1}}{p_{2}-q_{1}} & \frac{p_{2}-q_{2}}{p_{2}-q_{1}} \\
\frac{p_{1}-q_{1}}{p_{2}-q_{1}} & \frac{p_{2}-p_{1}}{p_{2}-q_{1}}
\end{array}\right)\left(\begin{array}{l}
u_{1, \text { in }} \\
u_{2, \text { in }}
\end{array}\right),
$$

which determines a Yang-Baxter map. Let

$$
\tilde{R}\left(p_{i}, q_{i} ; p_{j}, q_{j}\right):=\left(\begin{array}{ll}
\frac{q_{j}-q_{i}}{p_{j}-q_{i}} & \frac{p_{j}-q_{j}}{p_{j}-q_{i}} \\
\frac{p_{i}-q_{i}}{p_{j}-q_{i}} & \frac{p_{j}-p_{i}}{p_{j}-q_{i}}
\end{array}\right)
$$

act on the $i$ th and $j$ th slot of a direct sum. Then the Yang-Baxter equation holds. We note that $\tilde{R}\left(p_{i}, q_{i} ; p_{j}, q_{j}\right)=R\left(q_{i}, p_{i} ; q_{j}, p_{j}\right)^{\top}$.

\section{Pure 3-soliton solutions}

For $N=3$ we find

$$
\begin{aligned}
\tau= & \kappa_{11} \kappa_{22} \kappa_{33} \beta \mathrm{e}^{\vartheta_{111}}+\kappa_{11} \kappa_{22} \alpha_{12} \mathrm{e}^{\vartheta_{112}}+\kappa_{11} \kappa_{33} \alpha_{13} \mathrm{e}^{\vartheta_{121}} \\
& +\kappa_{22} \kappa_{33} \alpha_{23} \mathrm{e}^{\vartheta_{211}}+\kappa_{11} \mathrm{e}^{\vartheta_{122}}+\kappa_{22} \mathrm{e}^{\vartheta_{212}}+\kappa_{33} \mathrm{e}^{\vartheta_{221}}+\mathrm{e}^{\vartheta_{222}},
\end{aligned}
$$

where again $\kappa_{i j}=\eta_{i} K \xi_{j}$, and

$$
\begin{aligned}
\alpha_{i j}= & 1-\frac{\left(p_{i}-q_{i}\right)\left(p_{j}-q_{j}\right)}{\left(p_{i}-q_{j}\right)\left(p_{j}-q_{i}\right)} \frac{\kappa_{i j} \kappa_{j i}}{\kappa_{i i} \kappa_{j j}}, \\
\beta= & -2+\alpha_{12}+\alpha_{13}+\alpha_{23}+\frac{\left(p_{1}-q_{1}\right)\left(p_{2}-q_{2}\right)\left(p_{3}-q_{3}\right)}{\left(p_{1}-q_{3}\right)\left(p_{2}-q_{1}\right)\left(p_{3}-q_{2}\right)} \frac{\kappa_{12} \kappa_{23} \kappa_{31}}{\kappa_{11} \kappa_{22} \kappa_{33}} \\
& +\frac{\left(p_{1}-q_{1}\right)\left(p_{2}-q_{2}\right)\left(p_{3}-q_{3}\right)}{\left(p_{1}-q_{2}\right)\left(p_{2}-q_{3}\right)\left(p_{3}-q_{1}\right)} \frac{\kappa_{13} \kappa_{21} \kappa_{32}}{\kappa_{11} \kappa_{22} \kappa_{33}} .
\end{aligned}
$$

Furthermore, we obtain

$$
\begin{aligned}
F= & \left(\left(p_{1}-q_{1}\right) \alpha_{23} \kappa_{22} \kappa_{33} \xi_{1} \otimes \eta_{1}+\left(p_{2}-q_{2}\right) \alpha_{13} \kappa_{11} \kappa_{33} \xi_{2} \otimes \eta_{2}\right. \\
& +\left(p_{3}-q_{3}\right) \alpha_{12} \kappa_{11} \kappa_{22} \xi_{3} \otimes \eta_{3}+\frac{\left(p_{1}-q_{1}\right)\left(p_{2}-q_{2}\right)}{q_{1}-p_{2}} \alpha_{312} \kappa_{12} \kappa_{33} \xi_{1} \otimes \eta_{2} \\
& +\frac{\left(p_{1}-q_{1}\right)\left(p_{3}-q_{3}\right)}{q_{1}-p_{3}} \alpha_{213} \kappa_{13} \kappa_{22} \xi_{1} \otimes \eta_{3}+\frac{\left(p_{2}-q_{2}\right)\left(p_{3}-q_{3}\right)}{q_{2}-p_{3}} \alpha_{123} \kappa_{11} \kappa_{23} \xi_{2} \otimes \eta_{3}
\end{aligned}
$$




$$
\begin{aligned}
& +\frac{\left(p_{1}-q_{1}\right)\left(q_{2}-p_{2}\right)}{p_{1}-q_{2}} \alpha_{321} \kappa_{21} \kappa_{33} \xi_{2} \otimes \eta_{1}+\frac{\left(p_{1}-q_{1}\right)\left(q_{3}-p_{3}\right)}{p_{1}-q_{3}} \alpha_{231} \kappa_{22} \kappa_{31} \xi_{3} \otimes \eta_{1} \\
& \left.+\frac{\left(p_{2}-q_{2}\right)\left(q_{3}-p_{3}\right)}{p_{2}-q_{3}} \alpha_{132} \kappa_{11} \kappa_{32} \xi_{3} \otimes \eta_{2}\right) \mathrm{e}^{\vartheta_{111}} \\
& +\left(\left(p_{1}-q_{1}\right) \kappa_{22} \xi_{1} \otimes \eta_{1}+\left(p_{2}-q_{2}\right) \kappa_{11} \xi_{2} \otimes \eta_{2}+\frac{\left(p_{1}-q_{1}\right)\left(p_{2}-q_{2}\right)}{q_{1}-p_{2}} \kappa_{12} \xi_{1} \otimes \eta_{2}\right. \\
& \left.-\frac{\left(p_{1}-q_{1}\right)\left(p_{2}-q_{2}\right)}{p_{1}-q_{2}} \kappa_{21} \xi_{2} \otimes \eta_{1}\right) \mathrm{e}^{\vartheta_{112}} \\
& +\left(\left(p_{1}-q_{1}\right) \kappa_{33} \xi_{1} \otimes \eta_{1}+\left(p_{3}-q_{3}\right) \kappa_{11} \xi_{3} \otimes \eta_{3}+\frac{\left(p_{1}-q_{1}\right)\left(p_{3}-q_{3}\right)}{q_{1}-p_{3}} \kappa_{13} \xi_{1} \otimes \eta_{3}\right. \\
& \left.-\frac{\left(p_{1}-q_{1}\right)\left(p_{3}-q_{3}\right)}{p_{1}-q_{3}} \kappa_{31} \xi_{3} \otimes \eta_{1}\right) \mathrm{e}^{\vartheta_{121}} \\
& +\left(\left(p_{2}-q_{2}\right) \kappa_{33} \xi_{2} \otimes \eta_{2}+\left(p_{3}-q_{3}\right) \kappa_{22} \xi_{3} \otimes \eta_{3}+\frac{\left(p_{2}-q_{2}\right)\left(p_{3}-q_{3}\right)}{q_{2}-p_{3}} \kappa_{23} \xi_{2} \otimes \eta_{3}\right. \\
& \left.-\frac{\left(p_{2}-q_{2}\right)\left(p_{3}-q_{3}\right)}{p_{2}-q_{3}} \kappa_{32} \xi_{3} \otimes \eta_{2}\right) \mathrm{e}^{\vartheta_{211}} \\
& +\left(p_{1}-q_{1}\right) \xi_{1} \otimes \eta_{1} \mathrm{e}^{\vartheta_{122}}+\left(p_{2}-q_{2}\right) \xi_{2} \otimes \eta_{2} \mathrm{e}^{\vartheta_{212}}+\left(p_{3}-q_{3}\right) \xi_{3} \otimes \eta_{3} \mathrm{e}^{\vartheta_{221}},
\end{aligned}
$$

where

$$
\alpha_{k i j}=1-\frac{\left(q_{i}-p_{j}\right)\left(q_{k}-p_{k}\right) \kappa_{i k} \kappa_{k j}}{\left(q_{i}-p_{k}\right)\left(q_{k}-p_{j}\right) \kappa_{i j} \kappa_{k k}} .
$$

Note that $\alpha_{i j}=\alpha_{i j j}$. Recall that the coefficient of $\mathrm{e}^{\vartheta_{a b c}}$ in the expression for $\tau$, respectively, $F$, has been named $\mu_{a b c}$, respectively, $M_{a b c}$. The tropical value in the region where $\vartheta_{a b c}$ dominates all other phases is given by

$$
\phi_{a b c}=\frac{M_{a b c}}{\mu_{a b c}} .
$$

The corresponding values can be read off from (6.1) and (6.2).

The Yang-Baxter property of the nonlinear map (5.4) can be deduced from the pure 3-soliton solution in the following way. Numbering the (in $y$ direction) incoming solitons by 1,2, 3 in $x$ direction, for $t \ll 0$ first (according to increasing values of y) solitons 1 and 2 interact, then solitons 1 and 3 , and finally solitons 2 and 3 . For $t \gg 0$ solitons 2 and 3 meet first, then solitons 1 and 3, and finally solitons 1 and 2 . Also see Fig. 3 below. Recalling that the polarizations along the tropical limit graph do not depend on the variables $x, y, t$, this implies that in both cases we obtain the same outgoing polarizations. Hence, the Yang-Baxter equation (5.5) holds. This is worked out in detail only for the simpler vector KP case in the next subsection. But we checked the general case as well.

That we can check the Yang-Baxter equation in this way is because of the fact that, in the tropical limit and at fixed $t$, we have well-defined interaction points of solitons. In the original wave description, we can only compute the asymptotics, i.e., 

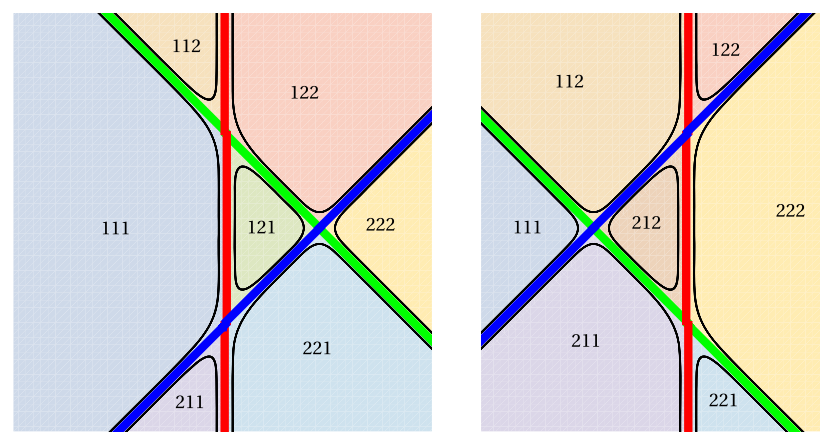

Fig. 3 Yang-Baxter relation in terms of vector KP line solitons. These are contour plots in the $x y$ plane (horizontal $x$ - and vertical $y$-axis) of a 3-soliton solution at negative, respectively, positive $t$. A number $a b c$ indicates the respective dominating phase region

the structure of incoming and outgoing solitons, but we have no description of what happens in the interaction region.

Because of the exponential localization of waves, a pure $N$-soliton solution, $N>2$, looks like a 2-soliton solution close enough to a crossing in the tropical limit graph. This becomes exact in the tropical limit. It implies that the Yang-Baxter map (5.4) acts at any crossing of the tropical limit graph. We will discuss this in more detail for the vector KP case in Sect. 7 .

\subsection{Pure vector KP 3-soliton solutions}

Now we restrict our considerations to the vector case $n=1$. Using

$$
\hat{\xi}_{i}=\frac{\xi_{i}}{K \xi_{i}}
$$

we obtain

$$
\begin{aligned}
& \hat{u}_{122,222}=\hat{\xi}_{1}, \quad \hat{u}_{212,222}=\hat{\xi}_{2}, \quad \hat{u}_{221,222}=\hat{\xi}_{3}, \\
& \hat{u}_{111,211}=\frac{\left(p_{1}-q_{2}\right)\left(p_{1}-q_{3}\right)}{\left(p_{1}-p_{2}\right)\left(p_{1}-p_{3}\right)} \hat{\xi}_{1}-\frac{\left(p_{2}-q_{2}\right)\left(p_{2}-q_{3}\right)}{\left(p_{1}-p_{2}\right)\left(p_{2}-p_{3}\right)} \hat{\xi}_{2}+\frac{\left(p_{3}-q_{2}\right)\left(p_{3}-q_{3}\right)}{\left(p_{1}-p_{3}\right)\left(p_{2}-p_{3}\right)} \hat{\xi}_{3}, \\
& \hat{u}_{111,121}=\frac{\left(p_{1}-q_{1}\right)\left(p_{1}-q_{3}\right)}{\left(p_{1}-p_{2}\right)\left(p_{1}-p_{3}\right)} \hat{\xi}_{1}-\frac{\left(p_{2}-q_{1}\right)\left(p_{2}-q_{3}\right)}{\left(p_{1}-p_{2}\right)\left(p_{2}-p_{3}\right)} \hat{\xi}_{2}+\frac{\left(p_{3}-q_{1}\right)\left(p_{3}-q_{3}\right)}{\left(p_{2}-p_{3}\right)\left(p_{1}-p_{3}\right)} \hat{\xi}_{3}, \\
& \hat{u}_{111,112}=\frac{\left(p_{1}-q_{1}\right)\left(p_{1}-q_{2}\right)}{\left(p_{1}-p_{2}\right)\left(p_{1}-p_{3}\right)} \hat{\xi}_{1}-\frac{\left(p_{2}-q_{1}\right)\left(p_{2}-q_{2}\right)}{\left(p_{1}-p_{2}\right)\left(p_{2}-p_{3}\right)} \hat{\xi}_{2}+\frac{\left(p_{3}-q_{1}\right)\left(p_{3}-q_{2}\right)}{\left(p_{1}-p_{3}\right)\left(p_{2}-p_{3}\right)} \hat{\xi}_{3}, \\
& \hat{u}_{121,221}=\frac{p_{1}-q_{3}}{p_{1}-p_{3}} \hat{\xi}_{1}-\frac{p_{3}-q_{3}}{p_{1}-p_{3}} \hat{\xi}_{3}, \quad \hat{u}_{121,122}=\frac{p_{1}-q_{1}}{p_{1}-p_{3}} \hat{\xi}_{1}-\frac{p_{3}-q_{1}}{p_{1}-p_{3}} \hat{\xi}_{3}, \\
& \hat{u}_{112,122}=\frac{p_{1}-q_{1}}{p_{1}-p_{2}} \hat{\xi}_{1}-\frac{p_{2}-q_{1}}{p_{1}-p_{2}} \hat{\xi}_{2}, \quad \hat{u}_{211,221}=\frac{p_{2}-q_{3}}{p_{2}-p_{3}} \hat{\xi}_{2}-\frac{p_{3}-q_{3}}{p_{2}-p_{3}} \hat{\xi}_{3}, \\
& \hat{u}_{211,212}=\frac{p_{2}-q_{2}}{p_{2}-p_{3}} \hat{\xi}_{2}-\frac{p_{3}-q_{2}}{p_{2}-p_{3}} \hat{\xi}_{3}, \quad \hat{u}_{112,212}=\frac{p_{1}-q_{2}}{p_{1}-p_{2}} \hat{\xi}_{1}-\frac{p_{2}-q_{2}}{p_{1}-p_{2}} \hat{\xi}_{2} .
\end{aligned}
$$


The contour plots in Fig. 3 show the structure at fixed $t$ with $t<0$ and $t>0$, respectively. The lines extending to the bottom are numbered by 1, 2, 3 from left to right (displayed as blue, red, green, respectively). Thinking of three particles undergoing a scattering process in $y$ direction, they carry polarizations that change at crossings. As $y$ increases we have

$$
\begin{aligned}
& \left(\hat{u}_{111,211}, \hat{u}_{211,221}, \hat{u}_{221,222}\right) \stackrel{12}{\mapsto}\left(\hat{u}_{121,221}, \hat{u}_{111,121}, \hat{u}_{221,222}\right) \\
& \quad \stackrel{13}{\mapsto}\left(\hat{u}_{122,222}, \hat{u}_{111,121}, \hat{u}_{121,122}\right) \stackrel{23}{\mapsto}\left(\hat{u}_{122,222}, \hat{u}_{112,122}, \hat{u}_{111,112}\right)
\end{aligned}
$$

for $t<0$, where the $i$ th entry contains the polarization of the $i$ th particle, and

$$
\begin{aligned}
& \left(\hat{u}_{111,211}, \hat{u}_{211,221}, \hat{u}_{221,222}\right) \stackrel{23}{\mapsto}\left(\hat{u}_{111,211}, \hat{u}_{212,222}, \hat{u}_{211,212}\right) \\
& \quad \stackrel{13}{\mapsto}\left(\hat{u}_{112,212}, \hat{u}_{212,222}, \hat{u}_{111,112}\right) \stackrel{12}{\mapsto}\left(\hat{u}_{122,222}, \hat{u}_{112,122}, \hat{u}_{111,112}\right)
\end{aligned}
$$

for $t>0$. The numbers $i j$ assigned to the steps refer to the "particles" involved. In both cases, we start and end with the same vectors, and this implies the Yang-Baxter equation for the associated transformations. Let $V_{a_{1} a_{2} a_{3}, b_{1} b_{2} b_{3}}$ be the column vector formed by the coefficients of $\hat{u}_{a_{1} a_{2} a_{3}, b_{1} b_{2} b_{3}}$ with respect to $\hat{\xi}_{1}, \hat{\xi}_{2}, \hat{\xi}_{3}$. The following matrices are composed of these column vectors,

$$
\begin{aligned}
& U_{123}=\left(\begin{array}{llll}
V_{111,211} & V_{211,221} & V_{221,222}
\end{array}\right), \quad U_{213}=\left(\begin{array}{lll}
V_{121,221} & V_{111,121} & V_{221,222}
\end{array}\right), \\
& U_{231}=\left(\begin{array}{llll}
V_{122,222} & V_{111,121} & V_{121,122}
\end{array}\right), \quad U_{321}=\left(\begin{array}{llll}
V_{122,222} & V_{112,122} & V_{111,112}
\end{array}\right), \\
& U_{132}=\left(\begin{array}{llll}
V_{111,211} & V_{212,222} & V_{211,212}
\end{array}\right), \quad U_{312}=\left(\begin{array}{lll}
V_{112,212} & V_{212,222} & V_{111,112}
\end{array}\right) .
\end{aligned}
$$

They represent the triplets of polarizations constituting the above chains. Next we define matrices

$$
\begin{aligned}
r_{12}= & U_{123}^{-1} U_{213}=U_{312}^{-1} U_{321}, \quad r_{13}=U_{213}^{-1} U_{231}=U_{132}^{-1} U_{312}, \\
& r_{23}=U_{231}^{-1} U_{321}=U_{123}^{-1} U_{132},
\end{aligned}
$$

which turn out to be given in terms of the $R$-matrix (5.6). For example,

$$
r_{13}=\left(\begin{array}{ccc}
\frac{p_{1}-p_{3}}{p_{1}-q_{3}} & 0 & \frac{p_{1}-q_{1}}{p_{1}-q_{3}} \\
0 & 1 & 0 \\
\frac{p_{3}-q_{3}}{p_{1}-q_{3}} & 0 & \frac{q_{1}-q_{3}}{p_{1}-q_{3}}
\end{array}\right)
$$

The Yang-Baxter equation reads

$$
r_{12} r_{13} r_{23}=r_{23} r_{13} r_{12}
$$

Figure 4 shows plots of $K u$ for a choice of the parameters. 

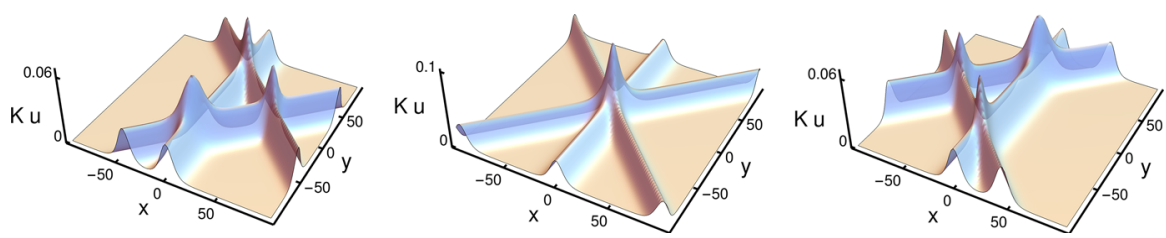

Fig. 4 Plots of the scalar $K u$ for a "Yang-Baxter line soliton configuration" of a vector KP equation at times $t<0, t=0$ and $t>0$

\subsection{Vector KdV 3-soliton solutions}

We impose the KdV reduction, see Remark 3.4, and replace (2.3) by

$$
\vartheta(P)=x P+t P^{3}+s P^{5}
$$

The additional last term introduces the next evolution variable $s$ of the KdV hierarchy, also see Remark 2.1 .

Let us consider, for simplicity, the $\operatorname{KdV}_{K}$ equation with $m=3$ and $K=(1,1,1)$, and the special solution with parameters

$$
\theta_{1}=I_{3}, \quad \chi_{1}=(1,-1,1)^{\top}
$$

The tropical limit graph is displayed in Fig. 5 for $p_{1}=1 / 2, p_{2}=3 / 4, p_{3}=1$, and different values of $s$. We have the matrix

$$
U_{123}=\left(\begin{array}{lll}
\hat{u}_{111,211} & \hat{u}_{211,221} & \hat{u}_{221,222}
\end{array}\right)=\left(\begin{array}{ccc}
\frac{\left(p_{1}+p_{2}\right)\left(p_{1}+p_{3}\right)}{\left(p_{1}-p_{2}\right)\left(p_{1}-p_{3}\right)} & 0 & 0 \\
-\frac{2 p_{2}\left(p_{2}+p_{3}\right)}{\left(p_{1}-p_{2}\right)\left(p_{2}-p_{3}\right)} & \frac{p_{2}+p_{3}}{p_{2}-p_{3}} & 0 \\
\frac{2 p_{3}\left(p_{2}+p_{3}\right)}{\left(p_{1}-p_{3}\right)\left(p_{2}-p_{3}\right)} & -\frac{2 p_{3}}{p_{2}-p_{3}} & 1
\end{array}\right)
$$
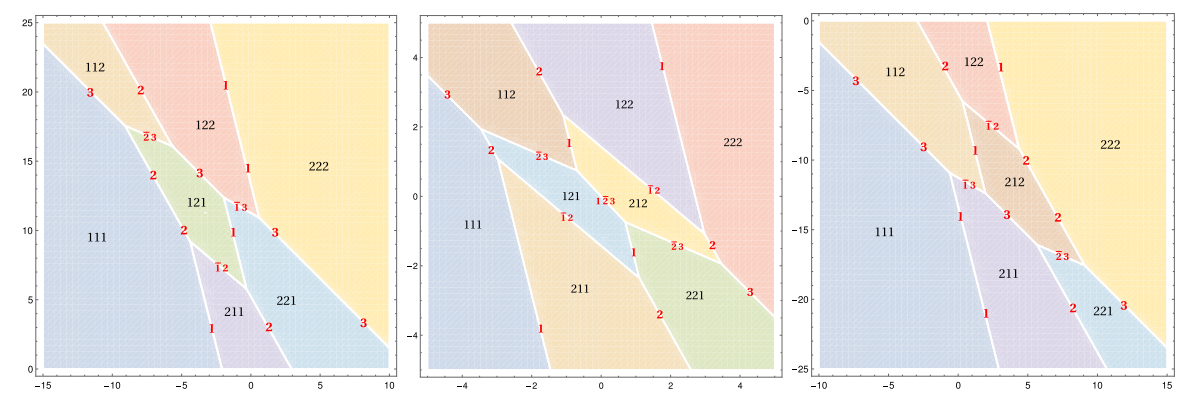

Fig. 5 Tropical limit graph and dominating phase regions of a vector KdV solution in two-dimensional space-time ( $x$ horizontal, $t$ vertical), at $s=-10, s=0$ and $s=10$. See Sect. 6.2. Numbers 1, 2, 3 (in red) attached to lines identify appearances of the respective soliton. Here bounded lines are formally associated with a pair of a (virtual) anti-soliton, indicated by a bar over the respective number, and a (virtual) soliton. At $s=0$, a "composite" of three virtual solitons (12̄3) shows up (color figure online) 
of initial polarizations. The next values $\hat{u}_{a b c \text {, def }}$ are then obtained by application of the $R$-matrix (5.7) from the right, and so forth, following either the left or the right graph in Fig. 5 in upwards (i.e., $t$ ) direction. Since the initial and the final polarizations are the same, the Yang-Baxter equation holds. Here the $R$-matrix describes the time evolution of polarizations in the tropical limit.

Figure 5 suggests to think of $R$-matrices as being associated with bounded lines, which may be thought of as representing "virtual solitons". Interaction of two solitons then means exchange of a virtual soliton, frequently called a "resonance".

At $s=0$, see the plot in the middle of Fig. 5, something peculiar occurs, namely a sort of three-particle interaction. This is a degenerate special case to which the YangBaxter description does not apply. To be precise, the statement that the Yang-Baxter map rules the polarizations along the tropical limit graph thus holds for $s \neq 0$.

To understand what this means, let us more generally think of any system, depending continuously on a parameter, say $s$, and carrying a structure, which is described by a map such that the left-hand side of the Yang-Baxter equation is realized for $s<0$, and the right-hand side for $s>0$. Then there is a "transition point", $s=0$, where the Yang-Baxter equation does not apply. The system is actually more complete, since it also contains a transition structure. The latter, however, cannot be resolved into a sequence of three applications of a map. This is the situation we meet in the tropical limit analysis of pure KP multi-soliton solutions. There are isolated parameter values, corresponding to "transitions", at which the Yang-Baxter equation does not apply.

\section{Tropical limit of pure vector solitons and the $\boldsymbol{R}$-matrix}

We set $n=1$ (vector case). The following results describe what happens at a crossing of two solitons, numbered by $i$ and $j$, depicted as a contour plot in Fig. 6.

\section{Lemma 7.1}

$$
\left(p_{i}-p_{j}\right) \phi_{I_{i j}(1,1)}+\left(q_{i}-q_{j}\right) \phi_{I_{i j}(2,2)}=\left(p_{i}-q_{j}\right) \phi_{I_{i j}(1,2)}+\left(q_{i}-p_{j}\right) \phi_{I_{i j}(2,1)} .
$$

Proof This is quickly verified for the 2 -soliton solution $(N=2)$. But at a crossing, a general solution $\phi$ is equivalent to a 2 -soliton solution, since there the four elementary

Fig. 6 A crossing of solitons with numbers $i$ and $j$ at fixed time in the $x y$ plane, and the numbers of the four phases that are involved

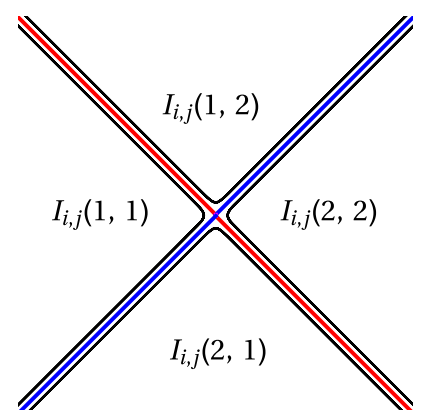


phases $\vartheta\left(p_{i, a}\right), \vartheta\left(p_{j, a}\right), a=1,2$, dominate all others, and hence, the exponential of any other phase vanishes in the tropical limit.

Remark 7.2 (7.1) can be regarded as a vector version of a scalar linear quadrilateral equation, satisfying "consistency on a cube", see (15) in [2]. Such a linear relation does not hold in the matrix case where $m, n>1$. But (5.1), with $\phi_{a b}$ replaced by $\phi_{I_{i j}(a, b)}$ (in which case we have $\phi_{I_{i j}(2,2)} \neq 0$, in general) is a nonlinear counterpart of (7.1). The latter can be deduced from it for $n=1$ by using (4.5).

\section{Theorem 7.3}

$$
\left(\hat{u}_{I_{i j}(1,1), I_{i j}(2,1)} \hat{u}_{I_{i j}(2,1), I_{i j}(2,2)}\right) R\left(p_{i}, q_{i} ; p_{j}, q_{j}\right)=\left(\hat{u}_{I_{i j}(1,2), I_{i j}(2,2)} \quad \hat{u}_{I_{i j}(1,1), I_{i j}(1,2)}\right)
$$

with

$$
R\left(p_{i}, q_{i} ; p_{j}, q_{j}\right)=\left(\begin{array}{ll}
\frac{p_{i}-p_{j}}{p_{i}-q_{j}} & \frac{p_{i}-q_{i}}{p_{i}-q_{j}} \\
\frac{p_{j}-q_{j}}{p_{i}-q_{j}} & \frac{q_{i}-q_{j}}{p_{i}-q_{j}}
\end{array}\right)
$$

Proof Using (4.4) we can directly verify that the following relations hold as a consequence of (7.1),

$$
\begin{aligned}
& \frac{p_{i}-p_{j}}{p_{i}-q_{j}} \hat{u}_{I_{i j}(1,1), I_{i j}(2,1)}+\frac{p_{j}-q_{j}}{p_{i}-q_{j}} \hat{u}_{I_{i j}(2,1), I_{i j}(2,2)}=\hat{u}_{I_{i j}(1,2), I_{i j}(2,2),} \\
& \frac{p_{i}-q_{i}}{p_{i}-q_{j}} \hat{u}_{I_{i j}(1,1), I_{i j}(2,1)}+\frac{q_{i}-q_{j}}{p_{i}-q_{j}} \hat{u}_{I_{i j}(2,1), I_{i j}(2,2)}=\hat{u}_{I_{i j}(1,1), I_{i j}(1,2) .}
\end{aligned}
$$

In matrix form, this is (7.2).

We already know that $R\left(p_{i}, q_{i} ; p_{j}, q_{j}\right)$ satisfies the Yang-Baxter equation.

\section{Construction of pure vector KP soliton solutions from a scalar KP solution and the $R$-matrix}

Given a $\tau$-function for a pure $N$-soliton solution of the scalar KP equation, the YangBaxter $R$-matrix found above can be used to construct a pure $N$-soliton solution of the vector KP equation. We will explain this for the case $N=3$. by

The $\tau$-function of the pure 3 -soliton solution of the scalar KP-II equation is given

$$
\tau=\sum_{a, b, c=1}^{2} \Delta_{a b c} \mathrm{e}^{\vartheta_{a b c}}, \quad \Delta_{a b c}=\left(p_{2, b}-p_{1, a}\right)\left(p_{3, c}-p_{1, a}\right)\left(p_{3, c}-p_{2, b}\right),
$$

as obtained by the Wronskian method (see, e.g., [13]). Comparison with (3.5) shows that $\mu_{I}=\Delta_{I}$. 

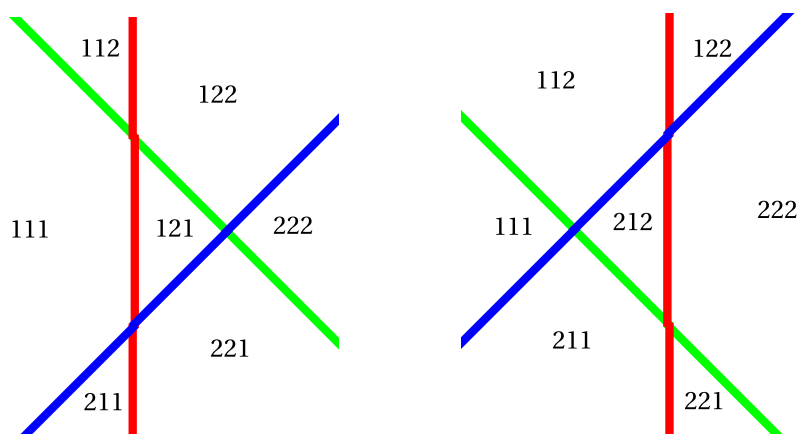

Fig. 7 Three-soliton configuration for $t<0$ and $t>0$, respectively, in the $x y$ plane. Numbers specify dominating phase regions

Starting at the bottom of both graphs in Fig. 7, we associate a column vector $\xi_{i}$ with the $i$ th soliton (counted from left to right) and normalize it such that $K \xi_{i}=1$. Accordingly, we set

$$
\hat{u}_{111,211}=\xi_{1}, \quad \hat{u}_{211,221}=\xi_{2}, \quad \hat{u}_{221,222}=\xi_{3} .
$$

By consecutive application of the $R$-matrix (5.6), we find the polarizations on the further line segments, proceeding in $y$ direction. There are different ways to proceed, but they are consistent since $R$ satisfies the Yang-Baxter equation. For example,

$$
\left(\begin{array}{ll}
\hat{u}_{121,221} \quad \hat{u}_{111,121}
\end{array}\right)=\left(\begin{array}{ll}
\left.\hat{u}_{111,211} \quad \hat{u}_{211,221}\right) R\left(p_{1}, q_{1} ; p_{2}, q_{2}\right)
\end{array}\right.
$$

This leads to

$$
\begin{aligned}
& \hat{u}_{121,221}=\frac{p_{1}-p_{2}}{p_{1}-q_{2}} \xi_{1}+\frac{p_{2}-q_{2}}{p_{1}-q_{2}} \xi_{2}, \quad \hat{u}_{111,121}=\frac{p_{1}-q_{1}}{p_{1}-q_{2}} \xi_{1}+\frac{q_{1}-q_{2}}{p_{1}-q_{2}} \xi_{2}, \\
& \hat{u}_{212,222}=\frac{p_{2}-p_{3}}{p_{2}-q_{3}} \xi_{2}+\frac{p_{3}-q_{3}}{p_{2}-q_{3}} \xi_{3}, \quad \hat{u}_{211,212}=\frac{p_{2}-q_{2}}{p_{2}-q_{3}} \xi_{2}+\frac{q_{2}-q_{3}}{p_{2}-q_{3}} \xi_{3}, \\
& \hat{u}_{122,222}=\frac{\left(p_{1}-p_{2}\right)\left(p_{1}-p_{3}\right)}{\left(p_{1}-q_{2}\right)\left(p_{1}-q_{3}\right)} \xi_{1}+\frac{\left(p_{1}-p_{3}\right)\left(p_{2}-q_{2}\right)}{\left(p_{1}-q_{2}\right)\left(p_{1}-q_{3}\right)} \xi_{2}+\frac{p_{3}-q_{3}}{p_{1}-q_{3}} \xi_{3}, \\
& \hat{u}_{121,122}=\frac{\left(p_{1}-p_{2}\right)\left(p_{1}-q_{1}\right)}{\left(p_{1}-q_{2}\right)\left(p_{1}-q_{3}\right)} \xi_{1}+\frac{\left(p_{1}-q_{1}\right)\left(p_{2}-q_{2}\right)}{\left(p_{1}-q_{2}\right)\left(p_{1}-q_{3}\right)} \xi_{2}+\frac{q_{1}-q_{3}}{p_{1}-q_{3}} \xi_{3}, \\
& \hat{u}_{111,112}=\frac{p_{1}-q_{1}}{p_{1}-q_{3}} \xi_{1}+\frac{\left(p_{2}-q_{2}\right)\left(q_{1}-q_{3}\right)}{\left(p_{1}-q_{3}\right)\left(p_{2}-q_{3}\right)} \xi_{2}+\frac{\left(q_{1}-q_{3}\right)\left(q_{2}-q_{3}\right)}{\left(p_{1}-q_{3}\right)\left(p_{2}-q_{3}\right)} \xi_{3}, \\
& \hat{u}_{112,212}=\frac{p_{1}-p_{3}}{p_{1}-q_{3}} \xi_{1}+\frac{\left(p_{2}-q_{2}\right)\left(p_{3}-q_{3}\right)}{\left(p_{1}-q_{3}\right)\left(p_{2}-q_{3}\right)} \xi_{2}+\frac{\left(p_{3}-q_{3}\right)\left(q_{2}-q_{3}\right)}{\left(p_{1}-q_{3}\right)\left(p_{2}-q_{3}\right)} \xi_{3}, \\
& \hat{u}_{112,122}=\frac{\left(p_{1}-p_{3}\right)\left(p_{1}-q_{1}\right)}{\left(p_{1}-q_{2}\right)\left(p_{1}-q_{3}\right)} \xi_{1}
\end{aligned}
$$




$$
\begin{aligned}
& +\frac{\left(p_{2}-p_{3}\right)\left(q_{1}-q_{2}\right)\left(p_{1}-q_{3}\right)+\left(p_{1}-q_{1}\right)\left(p_{2}-q_{2}\right)\left(p_{3}-q_{3}\right)}{\left(p_{1}-q_{3}\right)\left(p_{1}-q_{2}\right)\left(p_{2}-q_{3}\right)} \xi_{2} \\
& +\frac{\left(p_{3}-q_{3}\right)\left(q_{1}-q_{3}\right)}{\left(p_{1}-q_{3}\right)\left(p_{2}-q_{3}\right)} \xi_{3} .
\end{aligned}
$$

By "integration" of (4.4), we find $\phi_{a b c}$ up to a single constant. Setting $\phi_{222}=0$, we obtain

$$
\begin{aligned}
\phi_{111}= & \xi_{1}\left(p_{1}-q_{1}\right)+\xi_{2}\left(p_{2}-q_{2}\right)+\xi_{3}\left(p_{3}-q_{3}\right), \\
\phi_{112}= & \frac{\left(p_{1}-p_{3}\right)\left(p_{1}-q_{1}\right)}{p_{1}-q_{3}} \xi_{1}+\left(\frac{\left(q_{1}-q_{3}\right)\left(p_{2}-q_{2}\right)\left(p_{3}-q_{3}\right)}{\left(p_{1}-q_{3}\right)\left(q_{3}-p_{2}\right)}+p_{2}-q_{2}\right) \xi_{2} \\
& +\left(\frac{\left(q_{1}-q_{3}\right)\left(q_{2}-q_{3}\right)\left(p_{3}-q_{3}\right)}{\left(p_{1}-q_{3}\right)\left(q_{3}-p_{2}\right)}+p_{3}-q_{3}\right) \xi_{3} \\
\phi_{121}= & \frac{\left(p_{1}-p_{2}\right)\left(p_{1}-q_{1}\right)}{p_{1}-q_{2}} \xi_{1}+\frac{\left(p_{1}-q_{1}\right)\left(p_{2}-q_{2}\right)}{p_{1}-q_{2}} \xi_{2}+\left(p_{3}-q_{3}\right) \xi_{3}, \\
\phi_{211}= & \left(p_{2}-q_{2}\right) \xi_{2}+\left(p_{3}-q_{3}\right) \xi_{3}, \\
\phi_{122}= & \frac{\left(p_{1}-p_{2}\right)\left(p_{1}-p_{3}\right)\left(p_{1}-q_{1}\right)}{\left(p_{1}-q_{2}\right)\left(p_{1}-q_{3}\right)} \xi_{1}+\frac{\left(p_{1}-p_{3}\right)\left(p_{1}-q_{1}\right)\left(p_{2}-q_{2}\right)}{\left(p_{1}-q_{3}\right)} \xi_{2} \\
& +\frac{\left(p_{1}-q_{1}\right)\left(p_{3}-q_{3}\right)}{p_{1}-q_{3}} \xi_{3}, \\
\phi_{221}= & \left(p_{3}-q_{3}\right) \xi_{3}, \\
\phi_{212}= & \frac{\left(p_{2}-p_{3}\right)\left(p_{2}-q_{2}\right)}{p_{2}-q_{3}} \xi_{2}+\frac{\left(p_{2}-q_{2}\right)\left(p_{3}-q_{3}\right)}{p_{2}-q_{3}} \xi_{3} .
\end{aligned}
$$

From (4.1) we can read off $M_{a b c}$ and thus obtain via (3.2) and (3.4) the solution

$$
\phi=\frac{1}{\tau} \sum_{a, b, c=1}^{2} M_{a b c} \mathrm{e}^{\vartheta_{a b c}}
$$

of the vector pKP equation. This procedure can easily be applied to a larger number of solitons.

Remark 8.1 The construction described in this section cannot be extended to the matrix $\mathrm{KP}$ case, since then the function $\tau$ does not correspond to a solution of the scalar KP equation, or of any other meaningful equation.

\section{A solution of the tetrahedron equation}

In this section, we address again the case of three pure solitons, see Sect. 6. Because of the occurrence of phase shifts, in the tropical limit a crossing of two solitons does not really take place in a point. Figure 8 shows this for the second interaction (in the vertical $y$ direction) in Fig. 3, or Fig. 7. 


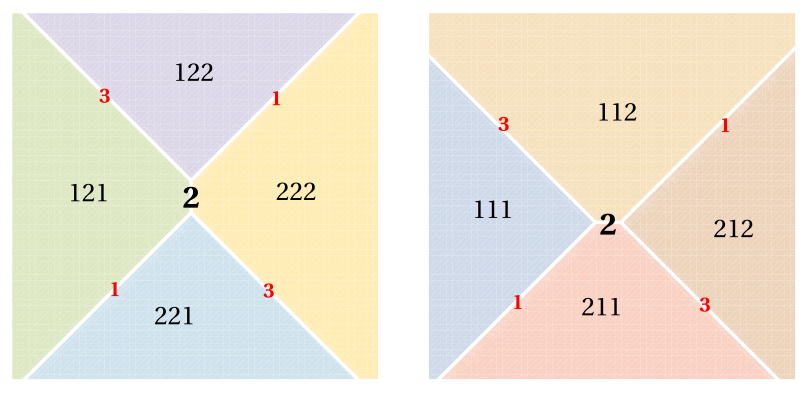

Fig. 8 Passing to the tropical limit and zooming into the second interaction "point" in the tropical limit of Fig. 3, shows the tropical origin of phase shifts. Again, the left figure refers to $t<0$, the right to $t>0$

We may think of associating with the additional edge in the left plot $(t<0)$ the polarization of the boundary between the phase regions numbered by $121=(1,2,1)$ and 222, and in the right plot $(t>0)$ that of the boundary between the phases 112 and 211. But this does not lead us to something meaningful. Instead, we make an educated guess and associate a mean value of vectors with an additional edge,

$$
\begin{aligned}
V_{1} & =\frac{1}{2}\left(V_{111,221}+V_{121,211}\right), \quad V_{2}=\frac{1}{2}\left(V_{121,222}+V_{122,221}\right), \\
V_{3} & =\frac{1}{2}\left(V_{111,122}+V_{121,112}\right),
\end{aligned}
$$

and

$$
\begin{aligned}
& \hat{V}_{1}=\frac{1}{2}\left(V_{112,222}+V_{122,212}\right), \quad \hat{V}_{2}=\frac{1}{2}\left(V_{111,212}+V_{112,211}\right), \\
& \hat{V}_{3}=\frac{1}{2}\left(V_{211,222}+V_{212,221}\right) .
\end{aligned}
$$

The inner boundary lines shown in the two plots in Fig. 8 are now associated with $V_{2}$, respectively, $\hat{V}_{2}$. In the first plot, the boundary between phase regions 122 and 221 is hidden, but there is also a polarization associated with it, namely $V_{122,221} . V_{2}$ is the mean value of the latter and $V_{121,222}$, which belongs to the visible inner boundary segment.

Now we have

$$
\begin{aligned}
S(i, j, k) & =\left.\left(\begin{array}{lll}
V_{1} & V_{2} & V_{3}
\end{array}\right)^{-1}\left(\begin{array}{lll}
\hat{V}_{1} & \hat{V}_{2} & \hat{V}_{3}
\end{array}\right)\right|_{p_{1} \mapsto p_{i}, p_{2} \mapsto p_{j}, p_{3} \mapsto p_{k}, q_{1} \mapsto q_{i}, q_{2} \mapsto q_{j}, q_{3} \mapsto q_{k}} \\
& =\frac{1}{\delta_{i j k}} \gamma^{i j k}-1_{3},
\end{aligned}
$$

where $\gamma^{i j k}=\left(\gamma_{r s}^{i j k}\right)$ with

$$
\begin{aligned}
& \gamma_{11}^{i j k}=\rho_{k}\left(\rho_{i}\left(p_{k}+q_{k}-2 q_{i}\right)+\rho_{j}\left(p_{k}+q_{k}-2 p_{j}\right)\right), \\
& \gamma_{22}^{i j k}=\rho_{j}\left(\rho_{i}\left(p_{j}+q_{j}-2 q_{i}\right)-\rho_{k}\left(p_{j}+q_{j}-2 q_{k}\right)\right),
\end{aligned}
$$




$$
\begin{aligned}
\gamma_{33}^{i j k} & =-\left(\rho_{i}\left(\rho_{j}\left(p_{i}+q_{i}-2 p_{j}\right)+\rho_{k}\left(p_{i}+q_{i}-2 q_{k}\right)\right),\right. \\
\gamma_{12}^{i j k} & =-\rho_{k} \frac{\rho_{i}^{2}-\rho_{j}^{2}}{\rho_{i}^{2}-\rho_{k}^{2}}\left(\rho_{k}^{2}-\rho_{i}\left(-2 q_{i}+p_{k}+q_{k}\right)\right), \\
\gamma_{13}^{i j k} & =-\rho_{k} \frac{\rho_{i}^{2}-\rho_{j}^{2}}{\rho_{j}^{2}-\rho_{k}^{2}}\left(\rho_{j}\left(p_{k}+q_{k}-2 p_{j}\right)+\rho_{k}^{2}\right), \\
\gamma_{21}^{i j k} & =-\rho_{j} \frac{\rho_{i}^{2}-\rho_{k}^{2}}{\rho_{i}^{2}-\rho_{j}^{2}}\left(\rho_{j}^{2}-\rho_{i}\left(p_{j}+q_{j}-2 q_{i}\right)\right), \\
\gamma_{23}^{i j k} & =\rho_{j} \frac{\rho_{i}^{2}-\rho_{k}^{2}}{\rho_{j}^{2}-\rho_{k}^{2}}\left(\rho_{j}^{2}-\rho_{k}\left(p_{j}+q_{j}-2 q_{k}\right)\right), \\
\gamma_{31}^{i j k} & =\rho_{i} \frac{\rho_{j}^{2}-\rho_{k}^{2}}{\rho_{i}^{2}-\rho_{j}^{2}}\left(\rho_{j}\left(p_{i}+q_{i}-2 p_{j}\right)+\rho_{i}^{2}\right), \\
\gamma_{32}^{i j k} & =\rho_{i} \frac{\rho_{j}^{2}-\rho_{k}^{2}}{\rho_{i}^{2}-\rho_{k}^{2}}\left(\rho_{i}^{2}-\rho_{k}\left(p_{i}+q_{i}-2 q_{k}\right)\right),
\end{aligned}
$$

and

$$
\delta_{i j k}=\left(p_{j}-q_{i}\right) \rho_{i} \rho_{j}+\left(q_{k}-q_{i}\right) \rho_{i} \rho_{k}+\left(q_{k}-p_{j}\right) \rho_{j} \rho_{k} .
$$

Here we set

$$
\rho_{i}=p_{i}-q_{i}
$$

Let $S_{\alpha \beta \gamma}(i, j, k)$ be the $6 \times 6$ matrix which acts via $S(i, j, k)$ on the positions $\alpha, \beta, \gamma$ of a 6 -component column vector, and as the identity on the others. Let

$$
\begin{aligned}
& \mathcal{R}_{123}=S_{123}(1,2,3), \quad \mathcal{R}_{145}=S_{145}(1,2,4), \quad \mathcal{R}_{246}=S_{246}(1,3,4), \\
& \mathcal{R}_{356}=S_{356}(2,3,4)
\end{aligned}
$$

We verified that this constitutes a (to our knowledge new) solution of the tetrahedron (Zamolodchikov) equation (see, e.g., [9] and references cited there)

$$
\mathcal{R}_{123} \mathcal{R}_{145} \mathcal{R}_{246} \mathcal{R}_{356}=\mathcal{R}_{356} \mathcal{R}_{246} \mathcal{R}_{145} \mathcal{R}_{123}
$$

An explanation for the choices of numbers in the above definition of $\mathcal{R}_{\alpha \beta \gamma}$ can be found in Fig. 9. Also see [15].

Remark 9.1 The $\mathrm{KdV}_{K}$ reduction $q_{i}=-p_{i}$ yields

$$
S_{\mathrm{KdV}}(i, j, k)=\left(\begin{array}{ccc}
-\frac{\left(p_{i}+p_{k}\right)\left(p_{j}-p_{k}\right)}{\left(p_{i}-p_{k}\right)\left(p_{j}+p_{k}\right)} & \frac{2\left(p_{i}-p_{j}\right) p_{k}}{\left(p_{i}-p_{k}\right)\left(p_{j}+p_{k}\right)} & \frac{2\left(p_{i}-p_{j}\right) p_{k}}{\left(p_{i}-p_{k}\right)\left(p_{j}+p_{k}\right)} \\
\frac{2 p_{j}\left(p_{i}+p_{k}\right)}{\left(p_{i}+p_{j}\right)\left(p_{j}+p_{k}\right)} & \frac{\left(p_{i}-p_{j}\right)\left(p_{j}-p_{k}\right)}{\left(p_{i}+p_{j}\right)\left(p_{j}+p_{k}\right)} & \frac{2 p_{j}\left(p_{i}+p_{k}\right)}{\left(p_{i}+p_{j}\right)\left(p_{j}+p_{k}\right)} \\
\frac{2 p_{i}\left(p_{j}-p_{k}\right)}{\left(p_{i}+p_{j}\right)\left(p_{i}-p_{k}\right)} & \frac{2 p_{i}\left(p_{j}-p_{k}\right)}{\left(p_{i}+p_{j}\right)\left(p_{i}-p_{k}\right)} & -\frac{\left(p_{i}-p_{j}\right)\left(p_{i}+p_{k}\right)}{\left(p_{i}+p_{j}\right)\left(p_{i}-p_{k}\right)}
\end{array}\right),
$$

which determines a simpler solution of the tetrahedron equation. 


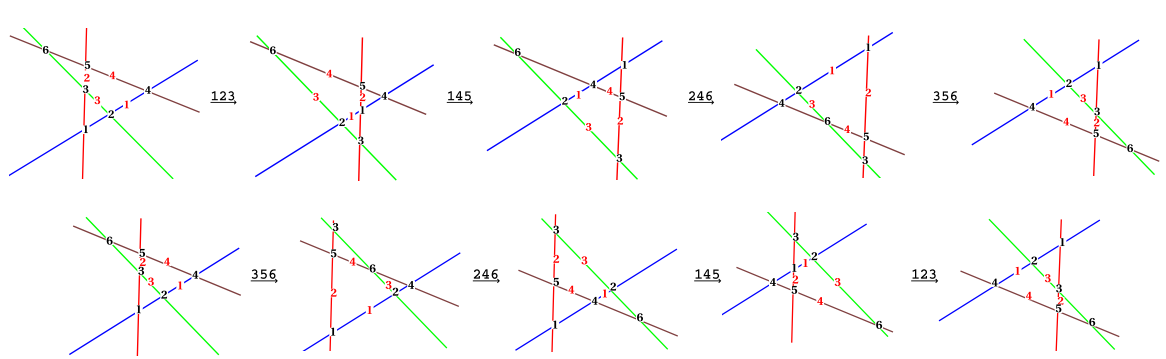

Fig. 9 KP line solitons remain parallel while moving. The two chains of line configurations (here shifts are disregarded) show the two different ways in which a 4-soliton solution can evolve from the same initial to the same final configuration. (The two chains represent the higher Bruhat order $B(4,2)$, cf. [9].) This implies the tetrahedron equation. Here (red) numbers attached to lines enumerate the four solitons. Also the crossings of pairs of them are enumerated (by numbers in black). Time evolution proceeds by inversion of triangles. In the first chain, the first step is the inversion of the triangle formed by the crossings numbered $1,2,3$. The second step is the inversion of the triangle formed by the crossings $1,4,5$. The latter involves the solitons with numbers 1, 2, 4 (color figure online)

\section{A generalization of the vector KP R-matrix and a solution of the functional tetrahedron equation}

The vector $\mathrm{KdV} R$-matrix (5.7) is obtained from the one-parameter $R$-matrix (see, e.g., $[15,20]$ for a similar $R$-matrix)

$$
R(x)=\left(\begin{array}{cc}
x & 1+x \\
1-x & -x
\end{array}\right)
$$

by setting $x=\left(p_{1}-p_{2}\right) /\left(p_{1}+p_{2}\right)$. The local Yang-Baxter equation

$$
R_{12}(x) R_{13}(y) R_{23}(z)=R_{23}(Z) R_{13}(Y) R_{12}(X),
$$

where indices $\alpha \beta$ indicate on which components of a threefold direct sum $R$ acts, determines the map $(x, y, z) \mapsto(X, Y, Z)$ given by

$$
X=\frac{x y}{x+z-x y z}, \quad Y=x+z-x y z, \quad Z=\frac{y z}{x+z-x y z} .
$$

A similar map appeared in [15,20]. A general argument (cf., e.g., [9] and references cited there) implies that

$$
\mathcal{R}(x, y, z):=(X, Y, Z)
$$

solves the (functional) tetrahedron equation (9.1), where a "product" of $\mathcal{R}$ 's now has to be interpreted as composition of maps. This tetrahedron map is involutive. Setting $x=\left(p_{1}-p_{2}\right) /\left(p_{1}+p_{2}\right), y=\left(p_{1}-p_{3}\right) /\left(p_{1}+p_{3}\right)$ and $z=\left(p_{2}-p_{3}\right) /\left(p_{2}+p_{3}\right)$, it becomes the identity. 
Correspondingly, the vector KP $R$-matrix (5.6) is obtained from the more general two-parameter $R$-matrix

$$
R(x, y)=\left(\begin{array}{cc}
x & y \\
1-x & 1-y
\end{array}\right)
$$

by setting $x=\left(p_{1}-p_{2}\right) /\left(p_{1}-q_{2}\right)$ and $y=\left(p_{1}-q_{1}\right) /\left(p_{1}-q_{2}\right)$. The local YangBaxter equation

$$
R_{12}(x, y) R_{13}(z, u) R_{23}(v, w)=R_{23}(V, W) R_{13}(Z, U) R_{12}(X, Y)
$$

determines the map $(x, y ; z, u ; v, w) \mapsto(X, Y ; Z, U ; V, W)$, where

$$
\begin{aligned}
& X=z C, \quad Y=\left(z-\frac{A}{x}\right) C, \quad Z=\frac{x}{C}, \\
& U=1-B, \quad V=\frac{v z(x-y)}{A}, \quad W=1-\frac{(1-u)(1-w)}{B},
\end{aligned}
$$

with

$$
\begin{aligned}
& A=u v x-u x-v y+x z, \quad B=u w x-u x-w y+1, \\
& C=\frac{A B-A(1-u)(1-w) x-B v(x-y)}{A B-A(1-u)(1-w)-B v z(x-y)} .
\end{aligned}
$$

Then

$$
\mathcal{R}(x, y ; z, u ; v, w):=(X, Y ; Z, U ; V, W)
$$

solves the functional (i.e., set-theoretical) tetrahedron equation.

\section{Conclusions}

In this work, we explored "pure" soliton solutions of matrix KP equations in a tropical limit. In case of the reduction to matrix $\mathrm{KdV}$, this consists of a planar graph in (two-dimensional) space-time, with polarizations assigned to its edges. Given initial polarizations, the evolution of them along the graph is ruled by a Yang-Baxter map. For the vector $\mathrm{KdV}$ equation, this is a linear map, hence an $R$ matrix. The classical scattering process of matrix $\mathrm{KdV}$ solitons resembles in the tropical limit the scattering of point particles in a 2-dimensional integrable quantum field theory, which is characterized by a scattering matrix that solves the (quantum) Yang-Baxter equation.

We have shown that all this holds more generally for $\mathrm{KP}_{K}$, where the tropical limit at a fixed time $t$ is given by a graph in the $x y$ plane, with polarizations attached to the soliton lines. Moreover, the vector KP case provides us with a realization of the "classical straight-string model" considered in [15]. It should be noticed, however, 
that KP line solitons in the tropical limit are not, in general, straight because of the appearance of (phase) shifts.

As a side product of our explorations of the tropical limit of pure vector KP solitons, we derived apparently new solutions of the tetrahedron (Zamolodchikov) equation. Whether these solutions are relevant, e.g., for the construction of solvable models of statistical mechanics in three dimensions, has still to be seen.

Another subclass of soliton solutions of the vector KP equation consists of those, for which the support at fixed time is a rooted and generically binary tree in the tropical limit. For the scalar KP equation, this has been extensively explored in [6,7]. Instead of the Yang-Baxter equation, the pentagon equation (see [9] and references therein) now plays a role in governing corresponding vector solitons. This is treated in a separate work [10].

Acknowledgements Open access funding provided by Max Planck Society. A.D. thanks V. Papageorgiou for a very helpful discussion.

Open Access This article is distributed under the terms of the Creative Commons Attribution 4.0 International License (http://creativecommons.org/licenses/by/4.0/), which permits unrestricted use, distribution, and reproduction in any medium, provided you give appropriate credit to the original author(s) and the source, provide a link to the Creative Commons license, and indicate if changes were made.

\section{References}

1. Ablowitz, M., Prinari, B., Trubatch, A.: Soliton interactions in the vector NLS equation. Inverse Probl. 20, 1217-1237 (2004)

2. Atkinson, J.: Linear quadrilateral lattice equations and multidimensional consistency. J. Phys. A Math. Theor. 42, 454005 (2009)

3. Biondini, G., Chakravarty, S.: Soliton solutions of the Kadomtsev-Petviashvili II equation. J. Math. Phys. 47, 033514-1-033514-26 (2006)

4. Chakravarty, S., Kodama, Y.: Classification of the line-soliton solutions of KPII. J. Phys. A Math. Theor. 41, 275209 (2008)

5. Chvartatskyi, O., Dimakis, A., Müller-Hoissen, F.: Self-consistent sources for integrable equations via deformations of binary Darboux transformations. Lett. Math. Phys. 106, 1139-1179 (2016)

6. Dimakis, A., Müller-Hoissen, F.: KP line solitons and Tamari lattices. J. Phys. A Math. Theor. 44, 025203 (2011)

7. Dimakis, A., Müller-Hoissen, F.: KP solitons, higher Bruhat and Tamari orders. In: Müller-Hoissen, F., Pallo, J., Stasheff, J. (eds.) Associahedra, Tamari Lattices and Related Structures, Progress in Mathematics, vol. 299, pp. 391-423. Birkhäuser, Basel (2012)

8. Dimakis, A., Müller-Hoissen, F.: KdV soliton interactions: a tropical view. J. Phys. Conf. Ser. 482, 012010 (2014)

9. Dimakis, A., Müller-Hoissen, F.: Simplex and polygon equations. SIGMA 11, 042 (2015)

10. Dimakis, A., Müller-Hoissen, F.: Matrix Kadomtsev-Petviashvili equation: tropical limit, Yang-Baxter and pentagon maps. Theor. Math. Phys. 196, 1164-1173 (2018)

11. Goncharenko, V.: Multisoliton solutions of the matrix KdV equation. Theor. Math. Phys. 126, 81-91 (2001)

12. Goncharenko, V., Veselov, A.: Yang-Baxter maps and matrix solitons. In: Shabat, A. (ed.) New Trends in Integrability and Partial Solvability, NATO Science Series II: Mathematics, Physics \& Chemistry, vol. 132, pp. 191-197. Kluwer, Dordrecht (2004)

13. Hirota, R.: The Direct Method in Soliton Theory, Cambridge Tracts in Mathematics, vol. 155. Cambridge University Press, Cambridge (2004)

14. Isojima, S., Willox, R., Satsuma, J.: Spider-web solutions of the coupled KP equation. J. Phys. A Math. Gen. 36, 9533-9552 (2003) 
15. Kashaev, R., Korepanov, I., Sergeev, S.: Functional tetrahedron equation. Theor. Math. Phys. 117, 1402-1403 (1998)

16. Kodama, Y.: KP Solitons and the Grassmannians. Springer Briefs in Mathematical Physics, vol. 22. Springer, Singapore (2017)

17. Kouloukas, T.: Relativistic collisions as Yang-Baxter maps. Phys. Lett. A 381, 3445-3449 (2017)

18. Maillet, J., Nijhoff, F.: Integrability for multidimensional lattice models. Phys. Lett. B 224, 389-396 (1989)

19. Maruno, K., Biondini, G.: Resonance and web structure in discrete soliton systems: the two-dimensional Toda lattice and its fully discrete and ultra-discrete analogues. J. Phys. A Math. Gen. 37, 11819-11839 (2004)

20. Sergeev, S.: Solutions of the functional tetrahedron equation connected with the local Yang-Baxter equation for the ferro-electric condition. Lett. Math. Phys. 45, 113-119 (1998)

21. Suris, Y., Veselov, A.: Lax matrices for Yang-Baxter maps. J. Nonlinear Math. Phys. 10, 223-230 (2003)

22. Tokihiro, S., Takahashi, D., Matsukidaira, J., Satsuma, J.: From soliton equations to integrable cellular automata through a limiting procedure. Phys. Rev. Lett. 76, 3247-3250 (1996)

23. Tsuchida, T.: $N$-soliton collision in the Manakov model. Progr. Theor. Phys. 111, 151-182 (2004)

24. Veselov, A.: Yang-Baxter maps and integrable dynamics. Phys. Lett. A 314, 214-221 (2003) 